\title{
Plant Based High Energy Mash Diets Supplemented With Nahco3, L-arginine+ Vitamin-c and Vegetable Oils Are Effective Against Tachycardia and Polycythemia in Broiler Chicken
}

\author{
Md Emran Hossain ( $\nabla$ emrancvasu@yahoo.com ) \\ Chittagong Veterinary and Animal Sciences University https://orcid.org/0000-0002-1750-7284 \\ Nasima Akter \\ Chittagong Veterinary and Animal Science University
}

\section{Research Article}

Keywords: Broiler, cardio-morphometry, performance, polycythemia, tachycardia

Posted Date: July 19th, 2021

DOl: https://doi.org/10.21203/rs.3.rs-709399/v1

License: (c) (i) This work is licensed under a Creative Commons Attribution 4.0 International License. Read Full License 


\section{Abstract}

The study aimed to investigate if plant based high energy mash diets supplemented with NaHCO3, L-arginine + vitamin-C and vegetable oils were effective against tachycardia and polycythemia in the commercial broiler chicken. Total 256Ross-308 day old male broiler chicks were randomly distributed into eight dietary treatment groups in a three way $2^{3}$ factorial arrangements (Three factors, i.e., $\mathrm{NaHCO}_{3}$, L-arginine + vitamin-C and vegetable oil each with two levels, e.g., 0 and $0.1 \%$ for $\mathrm{NaHCO}_{3}$ and L-arginine + vitamin-C; 3 and $4 \%$ of vegetable oil supplemented with basal diet). Iso-caloric and iso-nitrogenous diets were formulated and supplied ad libitum. The average daily feed intake (ADFI), average daily gain (ADG), feed efficiency (FE), carcass characteristics, cardio-pulmonary morphometry, total protein (TP), hemoglobin $(\mathrm{Hb})$, triiodothyronine $\left(\mathrm{T}_{3}\right)$, incidence of tachycardia and polycythemia were examined up to $35 \mathrm{~d}$. Supplementation of $\mathrm{NaHCO}_{3}$ decreased $(p<0.001)$ the ADFI at 1-14 $d, 15-35 d, 1-35 d$, improved $(p<0.01)$ the FE at $1-14 d$ and increased $(p<0.05)$ the serum TP. Dietary L-arginine + vitamin-C decreased $(p<0.01)$ the heart rate without affecting the performance parameters, carcass characteristics and hemato-biochemical indices.

Supplementation of vegetable oil decreased $(p<0.01)$ the ADFI at 1-14d, 15-35 $d, 1-35 d$, increased $(p<0.01)$ the ADG at 1-14d, improved $(p<0.001)$ the FE at 1-14 $d$ and increased $(p<0.05)$ the heart rate, $\mathrm{Hb}$ and PCV. Further, $\mathrm{NaHCO}_{3}, \mathrm{~L}$-arginine + vitamin-C and vegetable oil synergistically interacted to decrease the left and right ventricular weight, RV:TV and increased the $T_{3}$ without affecting overall performance, carcass characteristics and hemato-biochemical indices. It was concluded that, plant based high energy mash diets are not susceptible to tachycardia and polycythemia. Addition of $\mathrm{NaHCO}_{3}$, L-arginine + vitamin-C ameliorate the propensity of tachycardia and polycythemia without deteriorating performance, carcass characteristics and hematobiochemical indices of the commercial broiler birds in a dose dependent manner.

\section{Introduction}

Tachycardia in broiler is a patho-physiological interplay between the lungs and the heart initiated by the terminal consequences of the excessively elevated blood pressure within the pulmonary circulation (Wideman et al. 2013; Khajali and Wideman 2016). A broiler chick initially weighing around $40 \mathrm{~g}$ at hatch is likely to attain more than $4000 \mathrm{~g}$ in 8 weeks. This elevated growth of almost 100 -fold in just 8 weeks cannot be sustained devoid of similar remarkable increases in the functional capability of the heart and lungs (Wideman et al., 2013). The cardiac output increases 100 fold in 8 weeks post hatch, ranging from $8 \mathrm{ml} / \mathrm{min}$ for a $40 \mathrm{~g}$ chick to around $800 \mathrm{ml} / \mathrm{min}$ for a broiler weighing $4 \mathrm{~kg}$ (Wideman 1999; Wideman et al. 2013). Furthermore, in the study of Decuypere et al. (2000) on the muscle fibre typology it was evident that the choice for increased breast meat yield did not result in a proportionate increase in the heart, blood and lung weight due to the relative independence of glycolytic white muscle to the requirement for their oxygen. Due to these discrepancies, pulmonary vascular capacity in broiler birds is restricted and only a bit able to cope with continuous increases in the cardiac yield (Wideman and Bottje, 1993; Wideman, 2000). Therefore, modern broiler birds are prone to initially tachycardia and later on to the progressive development of pulmonary hypertension syndrome (PHS).

The PHS stimulates the red blood cells racing too rapidly through the pulmonary vasculature. Thus, the RBCs cannot achieve full blood-gas equilibrium because of a short transit time at the gas exchange surfaces leading to incomplete diffusive exchange of $\mathrm{O}_{2}$ and $\mathrm{CO}_{2}$ (Henry and Fedde, 1970; Powell et al., 1985). This inadequate residence time, thus, causes blood exiting the lungs and to enter the systemic flow with a lower than the usual partial pressure of $\mathrm{O}_{2}$ leading to hypoxemia. In the systemic flow, hypoxemia evokes extensive arteriolar dilatation to raise the blood flow and reinstate ample $\mathrm{O}_{2}$ delivery to the organs as well as tissues (Wideman et al. 1996, 1997, 2000; Wideman 2000; Wideman and Tackett 2000; Ruiz-Feria and Wideman 2001). The systemic arteriolar vasodilatation lets blood to way out to the large arteries more quickly accompanied by increment in the rate at which venous blood comes back to the right ventricle. The rise in venous return and the commencement of systemic arteriolar hypotension automatically stimulate the heart to increase the cardiac output leading to tachycardia. Additionally, persistent hypoxemia stimulates hematopoietin and markedly increases the hematocrit (Burton et al., 1971; Julien et al., 1985).

Increased hematocrit results polycythemia which boosts up blood thickness and is the most important reasons of the increased resistance to the blood-flow that consequences in PHS(Snyder, 1971; Penney et al., 1988). High energy pellet diets provoke PHS by stimulating lipolysis and being deficient in protein and thereby arginine as well. Plant proteins, by virtue, are more susceptible 
to be deficient in critical amino acids, i.e., lysine, methionine and arginine. Consequently, we warned that plant based high energy mash diet may prone to reduce the performance of the birds. We therefore, aimed to justify if plant based high energy mash diet supplemented with L-arginine + vitamin-C and sodium bicarbonate was effective against tachycardia and polycythemia in commercial broiler birds.

\section{Materials And Methods}

\section{Study design, animals and housing}

The experiment was conducted in a three way $2^{3}$ factorial arrangements (Three dietary factors, i.e., $\mathrm{NaHCO}_{3}$, L-arginine + vitamin$\mathrm{C}$ and vegetable oil each with two levels, e.g., $\mathrm{NaHCO}_{3}, 0$ and $0.1 \%$; L-arginine + vitamin-C, 0 and $0.1 \%$; Vegetable oil, 3 and $4 \%$ of the basal diet). Total 256 Ross-308 day old male broiler chicks were randomly distributed into eight dietary treatment groups with four replicates having 8 birds per pen (Table 1). The chicks were purchased from Nahar Agro Ltd., Chattogram, Bangladesh. All chicks were examined for male, grade $A$, uniform size without abnormalities. Floor space for each bird was 0.17 square feet in brooding box and 1 square feet in the cage. The birds were exposed to continuous lighting. The chicks were brooded at a temperature of $95^{\circ} \mathrm{F}, 90^{\circ} \mathrm{F}, 85^{\circ} \mathrm{F}$ and $80^{\circ} \mathrm{F}$ for the $1 \mathrm{st}, 2 \mathrm{nd}, 3 \mathrm{rd}$ and 4th weeks, respectively with the help of incandescent bulbs. Room temperature and humidity were measured by using wall mounted indoor analog thermo-hygrometer. Before arrival of the chicks, the shed was thoroughly cleaned and washed by using tap water with caustic soda. For disinfection, phenyl solution ( $1 \%$ $\mathrm{v} / \mathrm{v}$ ) was sprayed on the floor, corners and ceiling. Following spray, cleaning was done by using brush and clean water. Brooding boxes, rearing cages and pens were cleaned in the same manner. After cleaning and disinfection, the house was left empty one week for proper drying. After drying, all doors and windows was closed. The room was fumigated with single strength fumigant (Adding $40 \mathrm{ml}$ formalin to $20 \mathrm{~g} \mathrm{KMnO}_{4}$ for 100 cubic feet area) and sealed for 24 hours. On the next day, lime was spread on the floor and around the shed. Footbath containing potassium permanganate $(1 \% \mathrm{w} / \mathrm{v})$ was kept at the entrance of the poultry shed and changed daily. Feeders and drinkers were cleaned and washed with Timsen ${ }^{\circledR}$ solution $(0.3 \% \mathrm{v} / \mathrm{v})$ daily. All birds were vaccinated against Newcastle and Gumboro disease with both the primary and booster doses.

Table 1

Design of the experiment

\begin{tabular}{|c|c|c|c|c|c|}
\hline \multicolumn{3}{|c|}{ Dietary treatments } & \multirow[t]{2}{*}{ No. of treatments } & \multirow[t]{2}{*}{ No. of replicates } & \multirow{2}{*}{$\begin{array}{l}\text { No. of } \\
\text { birds }\end{array}$} \\
\hline $\mathrm{NaHCO}_{3}(\%)$ & L-arginine + vitamin-C (\%) & $\begin{array}{l}\text { Vegetable oil } \\
(\%)\end{array}$ & & & \\
\hline 0 & 0 & 3 & 1 & 4 & $4 \times 8=32$ \\
\hline 0 & 0 & 4 & 1 & 4 & $4 \times 8=32$ \\
\hline 0 & 0.10 & 3 & 1 & 4 & $4 \times 8=32$ \\
\hline 0 & 0.10 & 4 & 1 & 4 & $4 \times 8=32$ \\
\hline 0.10 & 0 & 3 & 1 & 4 & $4 \times 8=32$ \\
\hline 0.10 & 0 & 4 & 1 & 4 & $4 \times 8=32$ \\
\hline 0.10 & 0.10 & 3 & 1 & 4 & $4 \times 8=32$ \\
\hline 0.10 & 0.10 & 4 & 1 & 4 & $4 \times 8=32$ \\
\hline Total & 2 & 2 & 8 & 32 & $8 \times 4 \times 8=256$ \\
\hline
\end{tabular}

Experimental diets

Feed ingredients were purchased from local market. During purchase, wholesomeness and date of expiry was checked. Dry mash feed was provided to the birds throughout the whole experimental period. Eight different types of starter (1-14 d) and finisher (15-35 d) diets (Table 2-3) were formulated and supplied to the birds. All rations were iso-caloric and iso-nitrogenous. Feed was 
prepared manually and supplied ad-libitum to the birds on round small feeder for 1-10 days. After 10th day, small round feeders and waterers were replaced by large linear feeders and bell drinkers. 
Table 2

Formulation of the starter diets $(1-14 \mathrm{~d})$ for the experimental broiler birds

\begin{tabular}{|c|c|c|c|c|c|c|c|c|}
\hline \multirow[t]{2}{*}{ Amount (g/kg) } & \multicolumn{8}{|c|}{ Dietary treatments ${ }^{1}$} \\
\hline & $\mathrm{N}_{0} \mathrm{~A}_{0} \mathrm{~V}_{3}$ & $\mathrm{~N}_{0} \mathrm{~A}_{1} \mathrm{~V}_{3}$ & $N_{1} A_{0} V_{3}$ & $N_{1} A_{1} V_{3}$ & $\mathrm{~N}_{0} \mathrm{~A}_{0} \mathrm{~V}_{4}$ & $\mathrm{~N}_{0} \mathrm{~A}_{1} \mathrm{~V}_{4}$ & $N_{1} A_{0} V_{4}$ & $N_{1} A_{1} V_{4}$ \\
\hline Maize & 57.75 & 57.75 & 57.75 & 57.75 & 56.58 & 56.58 & 56.58 & 56.58 \\
\hline Rice polish & 0.50 & 0.50 & 0.50 & 0.50 & 0.50 & 0.50 & 0.50 & 0.50 \\
\hline Soybean oil & 1.50 & 1.50 & 1.50 & 1.50 & 2.00 & 2.00 & 2.00 & 2.00 \\
\hline Soybean meal & 37.00 & 37.00 & 37.00 & 37.00 & 37.30 & 37.30 & 37.30 & 37.30 \\
\hline Limestone & 1.48 & 1.38 & 1.38 & 1.28 & 1.72 & 1.62 & 1.62 & 1.52 \\
\hline $\mathrm{DCP}$ & 0.80 & 0.80 & 0.80 & 0.80 & 0.80 & 0.80 & 0.80 & 0.80 \\
\hline L-Lysine & 0.12 & 0.12 & 0.12 & 0.12 & 0.20 & 0.20 & 0.20 & 0.20 \\
\hline DL-Methionine & 0.20 & 0.20 & 0.20 & 0.20 & 0.25 & 0.25 & 0.25 & 0.25 \\
\hline Vitamin premix ${ }^{2}$ & 0.25 & 0.25 & 0.25 & 0.25 & 0.25 & 0.25 & 0.25 & 0.25 \\
\hline $\mathrm{NaHCO}_{3}$ & 0.00 & 0.00 & 0.10 & 0.10 & 0.00 & 0.00 & 0.10 & 0.10 \\
\hline L-arginine ${ }^{3}$ & 0.00 & 0.05 & 0.00 & 0.05 & 0.00 & 0.05 & 0.00 & 0.05 \\
\hline Vitamin- $C^{4}$ & 0.00 & 0.05 & 0.00 & 0.05 & 0.00 & 0.05 & 0.00 & 0.05 \\
\hline Feedzyme $^{5}$ & 0.05 & 0.05 & 0.05 & 0.05 & 0.05 & 0.05 & 0.05 & 0.05 \\
\hline Emulsifier ${ }^{6}$ & 0.10 & 0.10 & 0.10 & 0.10 & 0.10 & 0.10 & 0.10 & 0.10 \\
\hline Common salt & 0.25 & 0.25 & 0.25 & 0.25 & 0.25 & 0.25 & 0.25 & 0.25 \\
\hline Total & 100.0 & 100.0 & 100.0 & 100.0 & 100.0 & 100.0 & 100.0 & 100.0 \\
\hline \multicolumn{9}{|l|}{ Calculated values $^{7}$} \\
\hline Metabolizable energy (kcal/kg) & 3000.74 & 3000.74 & 3000.74 & 3000.74 & 3004.38 & 3000.74 & 3000.74 & 3000.74 \\
\hline Crude protein & 22.00 & 22.00 & 22.00 & 22.00 & 22.01 & 22.00 & 22.00 & 22.00 \\
\hline Crude fibre & 3.42 & 3.42 & 3.42 & 3.42 & 3.41 & 3.42 & 3.42 & 3.42 \\
\hline Ether extract & 3.95 & 3.95 & 3.95 & 3.95 & 4.42 & 3.95 & 3.95 & 3.95 \\
\hline Calcium & 0.92 & 0.92 & 0.92 & 0.92 & 1.09 & 0.92 & 0.92 & 0.92 \\
\hline Phosphorus & 0.65 & 0.65 & 0.65 & 0.65 & 0.69 & 0.65 & 0.65 & 0.65 \\
\hline Available phosphorus & 0.36 & 0.36 & 0.36 & 0.36 & 0.40 & 0.36 & 0.36 & 0.36 \\
\hline Sodium & 0.01 & 0.01 & 0.04 & 0.04 & 0.01 & 0.01 & 0.04 & 0.04 \\
\hline Potassium & 0.91 & 0.91 & 0.91 & 0.91 & 0.92 & 0.91 & 0.91 & 0.91 \\
\hline Magnesium & 0.13 & 0.13 & 0.13 & 0.13 & 0.13 & 0.13 & 0.13 & 0.13 \\
\hline Manganese (mg/kg) & 80.23 & 80.23 & 80.23 & 80.23 & 80.29 & 80.23 & 80.23 & 80.23 \\
\hline Zinc (mg/kg) & 86.05 & 86.05 & 86.05 & 86.05 & 85.92 & 86.05 & 86.05 & 86.05 \\
\hline Copper (mg/kg) & 17.49 & 17.49 & 17.49 & 17.49 & 17.51 & 17.49 & 17.49 & 17.49 \\
\hline Iron (mg/kg) & 156.30 & 156.30 & 156.30 & 156.30 & 156.36 & 156.30 & 156.30 & 156.30 \\
\hline
\end{tabular}




\begin{tabular}{|c|c|c|c|c|c|c|c|c|}
\hline \multirow[t]{2}{*}{ Amount (g/kg) } & \multicolumn{8}{|c|}{ Dietary treatments ${ }^{1}$} \\
\hline & $\mathrm{N}_{0} \mathrm{~A}_{0} \mathrm{~V}_{3}$ & $N_{0} A_{1} V_{3}$ & $N_{1} A_{0} V_{3}$ & $N_{1} A_{1} V_{3}$ & $\mathrm{~N}_{0} \mathrm{~A}_{0} \mathrm{~V}_{4}$ & $\mathrm{~N}_{0} \mathrm{~A}_{1} \mathrm{~V}_{4}$ & $N_{1} A_{0} V_{4}$ & $N_{1} A_{1} V_{4}$ \\
\hline Lysine & 1.31 & 1.31 & 1.31 & 1.31 & 1.39 & 1.31 & 1.31 & 1.31 \\
\hline Leucine & 1.88 & 1.88 & 1.88 & 1.88 & 1.87 & 1.88 & 1.88 & 1.88 \\
\hline Iso-leucine & 0.94 & 0.94 & 0.94 & 0.94 & 0.94 & 0.94 & 0.94 & 0.94 \\
\hline Valine & 1.04 & 1.04 & 1.04 & 1.04 & 1.04 & 1.04 & 1.04 & 1.04 \\
\hline Threonine & 1.21 & 1.21 & 1.21 & 1.21 & 1.22 & 1.21 & 1.21 & 1.21 \\
\hline Methionine & 0.54 & 0.54 & 0.54 & 0.54 & 0.59 & 0.54 & 0.54 & 0.54 \\
\hline Tryptophan & 0.27 & 0.27 & 0.27 & 0.27 & 0.27 & 0.27 & 0.27 & 0.27 \\
\hline Phenylalanine & 1.09 & 1.09 & 1.09 & 1.09 & 1.09 & 1.09 & 1.09 & 1.09 \\
\hline Cystine + methionine & 0.73 & 0.73 & 0.73 & 0.73 & 0.78 & 0.73 & 0.73 & 0.73 \\
\hline \multicolumn{9}{|l|}{ Analyzed values $(\%)^{8}$} \\
\hline AGE $(\mathrm{kcal} / \mathrm{kg})^{9}$ & 3513.96 & 3506.92 & 3510.44 & 3503.40 & 3496.35 & 3499.87 & 3492.83 & 3489.31 \\
\hline Crude protein & 21.56 & 21.12 & 20.90 & 21.34 & 21.78 & 21.56 & 21.12 & 21.56 \\
\hline Crude fibre & 3.35 & 3.28 & 3.25 & 3.32 & 3.39 & 3.35 & 3.28 & 3.35 \\
\hline Ether extract & 3.87 & 3.79 & 3.75 & 3.83 & 3.91 & 3.87 & 3.79 & 3.87 \\
\hline Calcium & 0.90 & 0.88 & 0.87 & 0.89 & 0.91 & 0.90 & 0.88 & 0.90 \\
\hline Phosphorus & 0.64 & 0.62 & 0.62 & 0.63 & 0.64 & 0.64 & 0.62 & 0.64 \\
\hline \multicolumn{9}{|c|}{${ }^{1} \mathrm{~N}_{0} \mathrm{~A}_{1} \mathrm{~V}_{3}=\mathrm{NaHCO}_{3} 0 \%$, L-arginine $0.05 \%+$ Vitamin-C $0.05 \%$, Vegetable oil $3 \%$} \\
\hline \multicolumn{9}{|c|}{$\mathrm{N}_{1} \mathrm{~A}_{0} \mathrm{~V}_{3}=\mathrm{NaHCO}_{3} 0.1 \%$, L-arginine $0 \%+$ Vitamin-C $0 \%$, vegetable oil $3 \%$} \\
\hline \multicolumn{9}{|c|}{$\mathrm{N}_{1} \mathrm{~A}_{1} \mathrm{~V}_{3}=\mathrm{NaHCO}_{3} 0.1 \%$, L-arginine $0.05 \%+$ Vitamin-C $0.05 \%$, Vegetable oil $3 \%$} \\
\hline \multicolumn{9}{|c|}{$\mathrm{N}_{0} \mathrm{~A}_{0} \mathrm{~V}_{4}=\mathrm{NaHCO}_{3} 0 \%$, L-arginine $0 \%+$ Vitamin-C $0 \%$, Vegetable oil $4 \%$} \\
\hline \multicolumn{9}{|c|}{$\mathrm{N}_{0} \mathrm{~A}_{1} \mathrm{~V}_{4}=\mathrm{NaHCO}_{3} \mathrm{O} \%$, L-arginine $0.05 \%+$ Vitamin-C $0.05 \%$, Vegetable oil $4 \%$} \\
\hline \multicolumn{9}{|c|}{$\mathrm{N}_{1} \mathrm{~A}_{0} \mathrm{~V}_{4}=\mathrm{NaHCO}_{3} 0.1 \%$, L-arginine $0 \%+$ Vitamin-C $0 \%$, Vegetable oil $4 \%$} \\
\hline \multicolumn{9}{|c|}{$\mathrm{N}_{1} \mathrm{~A}_{1} \mathrm{~V}_{4}=\mathrm{NaHCO}_{3} 0.1 \%$, L-arginine $0.05 \%+$ Vitamin-C $0.05 \%$, Vegetable oil $4 \%$} \\
\hline \multicolumn{9}{|c|}{ 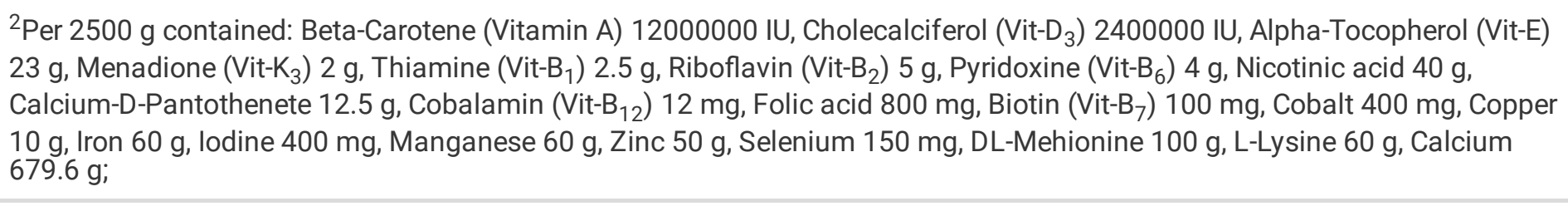 } \\
\hline \multicolumn{9}{|c|}{${ }^{3}$ L-Arginine 99.0\%, Vitamondo private limited, 89/19, Kamarajar colony, Musiri, TrichyTiruchirappalli TN 621211 India; } \\
\hline \multicolumn{9}{|c|}{${ }^{4}$ Vitamin-C 97\%, Sridhar Enterprises, Ayanambakkam, Chennai, Tamil Nadu, India; } \\
\hline
\end{tabular}




\section{Amount $(g / k g)$}

\section{Dietary treatments ${ }^{1}$}

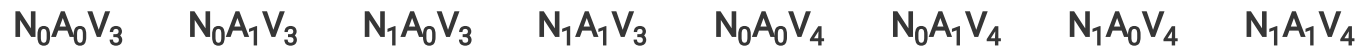

${ }^{6}$ Contained: Phosphatidyl-choline (PC) 16\%, Phosphatidyl-ethanolamine (PE) 10\%, Phosphatidic-acid (PA) 11\%, Phosphatidylinositol (PI) 8\%, Lyso-phosphatidyl-choline (LPC) 8\%, Lyso-phosphatidyl-ethanolamine (LPE) 13\%, Lyso-phosphatidic-acid (LPA) 6\%, Lyso-phosphatidyl-inositol (LPI) 8\%, Carrier 20\% (Brand-Molimen, Country of origin-Spain);

${ }^{7}$ Unit was considered as \% or otherwise stated;

${ }^{8}$ As per standard procedure (AOAC, 2019);

${ }^{9}$ Apparent gross energy $(\mathrm{kcal} / \mathrm{kg})$. 
Table 3

Formulation of the finisher diets $(15-35 \mathrm{~d})$ for the experimental broiler birds

\begin{tabular}{|c|c|c|c|c|c|c|c|c|}
\hline \multirow[t]{2}{*}{ Amount (g/kg) } & \multicolumn{8}{|c|}{ Dietary treatments ${ }^{1}$} \\
\hline & $\mathrm{N}_{0} \mathrm{~A}_{0} \mathrm{~V}_{3}$ & $\mathrm{~N}_{0} \mathrm{~A}_{1} \mathrm{~V}_{3}$ & $N_{1} A_{0} V_{3}$ & $N_{1} A_{1} V_{3}$ & $\mathrm{~N}_{0} \mathrm{~A}_{0} \mathrm{~V}_{4}$ & $\mathrm{~N}_{0} \mathrm{~A}_{1} \mathrm{~V}_{4}$ & $N_{1} A_{0} V_{4}$ & $N_{1} A_{1} V_{4}$ \\
\hline Maize & 60.00 & 60.00 & 60.00 & 60.00 & 56.00 & 56.00 & 56.00 & 56.00 \\
\hline Rice polish & 1.25 & 1.25 & 1.25 & 1.25 & 2.25 & 2.25 & 2.25 & 2.25 \\
\hline Soybean oil & 3.00 & 3.00 & 3.00 & 3.00 & 4.00 & 4.00 & 4.00 & 4.00 \\
\hline Soybean meal & 32.10 & 32.10 & 32.10 & 32.10 & 32.60 & 32.60 & 32.60 & 32.60 \\
\hline Limestone & 1.88 & 1.78 & 1.78 & 1.68 & 2.00 & 1.90 & 1.90 & 1.80 \\
\hline $\mathrm{DCP}$ & 0.80 & 0.80 & 0.80 & 0.80 & 1.95 & 1.95 & 1.95 & 1.95 \\
\hline L-Lysine & 0.12 & 0.12 & 0.12 & 0.12 & 0.25 & 0.25 & 0.25 & 0.25 \\
\hline DL-Methionine & 0.20 & 0.20 & 0.20 & 0.20 & 0.30 & 0.30 & 0.30 & 0.30 \\
\hline Vitamin premix ${ }^{2}$ & 0.25 & 0.25 & 0.25 & 0.25 & 0.25 & 0.25 & 0.25 & 0.25 \\
\hline $\mathrm{NaHCO}_{3}$ & 0.00 & 0.00 & 0.10 & 0.10 & 0.00 & 0.00 & 0.10 & 0.10 \\
\hline L-arginine ${ }^{3}$ & 0.00 & 0.05 & 0.00 & 0.05 & 0.00 & 0.05 & 0.00 & 0.05 \\
\hline Vitamin- $C^{4}$ & 0.00 & 0.05 & 0.00 & 0.05 & 0.00 & 0.05 & 0.00 & 0.05 \\
\hline Feedzyme $^{5}$ & 0.05 & 0.05 & 0.05 & 0.05 & 0.05 & 0.05 & 0.05 & 0.05 \\
\hline Emulsifier ${ }^{6}$ & 0.10 & 0.10 & 0.10 & 0.10 & 0.10 & 0.10 & 0.10 & 0.10 \\
\hline Common salt & 0.25 & 0.25 & 0.25 & 0.25 & 0.25 & 0.25 & 0.25 & 0.25 \\
\hline Total & 100.0 & 100.0 & 100.0 & 100.0 & 100.0 & 100.0 & 100.0 & 100.0 \\
\hline \multicolumn{9}{|l|}{ Calculated values $^{7}$} \\
\hline Metabolizable energy (kcal/kg) & 3102.26 & 3102.26 & 3102.26 & 3102.26 & 3101.38 & 3101.38 & 3101.38 & 3101.38 \\
\hline Crude protein & 20.02 & 20.02 & 20.02 & 20.02 & 20.03 & 20.03 & 20.03 & 20.03 \\
\hline Crude fibre & 3.23 & 3.23 & 3.23 & 3.23 & 3.26 & 3.26 & 3.26 & 3.26 \\
\hline Ether extract & 5.49 & 5.49 & 5.49 & 5.49 & 6.55 & 6.55 & 6.55 & 6.55 \\
\hline Calcium & 1.08 & 1.08 & 1.08 & 1.08 & 1.40 & 1.40 & 1.40 & 1.40 \\
\hline Phosphorus & 0.65 & 0.65 & 0.65 & 0.65 & 0.86 & 0.86 & 0.86 & 0.86 \\
\hline Available phosphorus & 0.37 & 0.37 & 0.37 & 0.37 & 0.57 & 0.57 & 0.57 & 0.57 \\
\hline Sodium & 0.01 & 0.01 & 0.04 & 0.04 & 0.01 & 0.01 & 0.04 & 0.04 \\
\hline Potassium & 0.80 & 0.80 & 0.80 & 0.80 & 0.83 & 0.83 & 0.83 & 0.83 \\
\hline Magnesium & 0.12 & 0.12 & 0.12 & 0.12 & 0.13 & 0.13 & 0.13 & 0.13 \\
\hline Manganese (mg/kg) & 79.76 & 79.76 & 79.76 & 79.76 & 81.89 & 81.89 & 81.89 & 81.89 \\
\hline Zinc (mg/kg) & 87.93 & 87.93 & 87.93 & 87.93 & 93.01 & 93.01 & 93.01 & 93.01 \\
\hline Copper (mg/kg) & 16.76 & 16.76 & 16.76 & 16.76 & 16.84 & 16.84 & 16.84 & 16.84 \\
\hline Iron (mg/kg) & 148.05 & 148.05 & 148.05 & 148.05 & 148.63 & 148.63 & 148.63 & 148.63 \\
\hline
\end{tabular}




\begin{tabular}{|c|c|c|c|c|c|c|c|c|}
\hline \multirow[t]{2}{*}{ Amount (g/kg) } & \multicolumn{8}{|c|}{ Dietary treatments ${ }^{1}$} \\
\hline & $\mathrm{N}_{0} \mathrm{~A}_{0} \mathrm{~V}_{3}$ & $N_{0} A_{1} V_{3}$ & $\mathrm{~N}_{1} \mathrm{~A}_{0} \mathrm{~V}_{3}$ & $N_{1} A_{1} V_{3}$ & $\mathrm{~N}_{0} \mathrm{~A}_{0} \mathrm{~V}_{4}$ & $\mathrm{~N}_{0} \mathrm{~A}_{1} \mathrm{~V}_{4}$ & $\mathrm{~N}_{1} \mathrm{~A}_{0} \mathrm{~V}_{4}$ & $\mathrm{~N}_{1} \mathrm{~A}_{1} \mathrm{~V}_{4}$ \\
\hline Lysine & 1.18 & 1.18 & 1.18 & 1.18 & 1.32 & 1.32 & 1.32 & 1.32 \\
\hline Leucine & 1.73 & 1.73 & 1.73 & 1.73 & 1.72 & 1.72 & 1.72 & 1.72 \\
\hline Iso-leucine & 0.85 & 0.85 & 0.85 & 0.85 & 0.86 & 0.86 & 0.86 & 0.86 \\
\hline Valine & 0.95 & 0.95 & 0.95 & 0.95 & 0.95 & 0.95 & 0.95 & 0.95 \\
\hline Threonine & 1.08 & 1.08 & 1.08 & 1.08 & 1.09 & 1.09 & 1.09 & 1.09 \\
\hline Methionine & 0.52 & 0.52 & 0.52 & 0.52 & 0.61 & 0.61 & 0.61 & 0.61 \\
\hline Tryptophan & 0.24 & 0.24 & 0.24 & 0.24 & 0.25 & 0.25 & 0.25 & 0.25 \\
\hline Phenylalanine & 0.99 & 0.99 & 0.99 & 0.99 & 0.99 & 0.99 & 0.99 & 0.99 \\
\hline Cystine + methionine & 0.71 & 0.71 & 0.71 & 0.71 & 0.80 & 0.80 & 0.80 & 0.80 \\
\hline \multicolumn{9}{|l|}{ Analyzed values $(\%)^{8}$} \\
\hline AGE $(\mathrm{kcal} / \mathrm{kg})^{9}$ & 3603.78 & 3596.56 & 3602.17 & 3592.95 & 3589.72 & 3592.95 & 3596.56 & 3589.33 \\
\hline Crude protein & 19.82 & 19.91 & 19.90 & 19.88 & 20.02 & 19.82 & 19.92 & 19.89 \\
\hline Crude fibre & 3.17 & 3.10 & 3.07 & 3.13 & 3.20 & 3.17 & 3.10 & 3.17 \\
\hline Ether extract & 5.38 & 5.27 & 5.22 & 5.33 & 5.44 & 5.38 & 5.27 & 5.38 \\
\hline Calcium & 1.06 & 1.04 & 1.03 & 1.05 & 1.07 & 1.06 & 1.04 & 1.06 \\
\hline Phosphorus & 0.64 & 0.62 & 0.62 & 0.63 & 0.64 & 0.64 & 0.62 & 0.64 \\
\hline \multicolumn{9}{|c|}{${ }^{1} \mathrm{~N}_{0} \mathrm{~A}_{1} \mathrm{~V}_{3}=\mathrm{NaHCO}_{3} 0 \%$, L-arginine $0.05 \%+$ Vitamin-C $0.05 \%$, Vegetable oil $3 \%$} \\
\hline \multicolumn{9}{|c|}{$\mathrm{N}_{1} \mathrm{~A}_{0} \mathrm{~V}_{3}=\mathrm{NaHCO}_{3} 0.1 \%$, L-arginine $0 \%+$ Vitamin-C $0 \%$, vegetable oil $3 \%$} \\
\hline \multicolumn{9}{|c|}{$\mathrm{N}_{1} \mathrm{~A}_{1} \mathrm{~V}_{3}=\mathrm{NaHCO}_{3} 0.1 \%$, L-arginine $0.05 \%+$ Vitamin-C $0.05 \%$, Vegetable oil $3 \%$} \\
\hline \multicolumn{9}{|c|}{$\mathrm{N}_{0} \mathrm{~A}_{0} \mathrm{~V}_{4}=\mathrm{NaHCO}_{3} 0 \%$, L-arginine $0 \%+$ Vitamin-C $0 \%$, Vegetable oil $4 \%$} \\
\hline \multicolumn{9}{|c|}{$\mathrm{N}_{0} \mathrm{~A}_{1} \mathrm{~V}_{4}=\mathrm{NaHCO}_{3} \mathrm{O} \%$, L-arginine $0.05 \%+$ Vitamin-C $0.05 \%$, Vegetable oil $4 \%$} \\
\hline \multicolumn{9}{|c|}{$\mathrm{N}_{1} \mathrm{~A}_{0} \mathrm{~V}_{4}=\mathrm{NaHCO}_{3} 0.1 \%$, L-arginine $0 \%+$ Vitamin-C $0 \%$, Vegetable oil $4 \%$} \\
\hline \multicolumn{9}{|c|}{$\mathrm{N}_{1} \mathrm{~A}_{1} \mathrm{~V}_{4}=\mathrm{NaHCO}_{3} 0.1 \%$, L-arginine $0.05 \%+$ Vitamin-C $0.05 \%$, Vegetable oil $4 \%$} \\
\hline \multicolumn{9}{|c|}{ 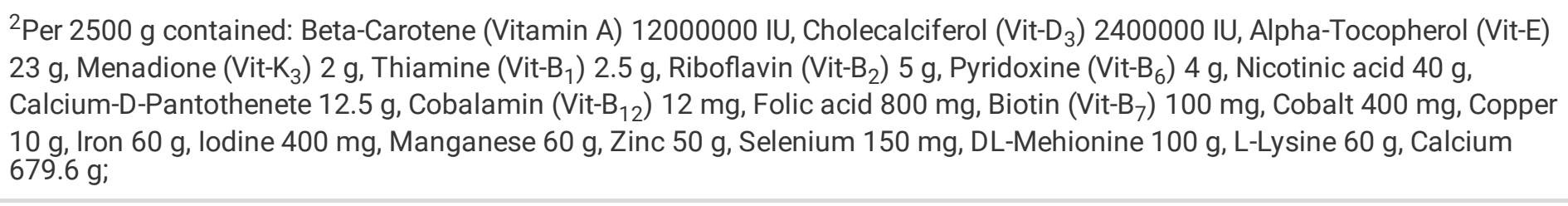 } \\
\hline \multicolumn{9}{|c|}{ 32L-Arginine 99.0\%, Vitamondo private limited, 89/19, Kamarajar colony, Musiri, TrichyTiruchirappalli TN 621211 India; } \\
\hline \multicolumn{9}{|c|}{${ }^{4}$ Vitamin-C 97\%, Sridhar Enterprises, Ayanambakkam, Chennai, Tamil Nadu, India; } \\
\hline
\end{tabular}




\begin{tabular}{|c|c|c|c|c|c|c|c|c|}
\hline \multirow[t]{2}{*}{ Amount $(\mathrm{g} / \mathrm{kg})$} & \multicolumn{8}{|c|}{ Dietary treatments ${ }^{1}$} \\
\hline & $\mathrm{N}_{0} \mathrm{~A}_{0} \mathrm{~V}_{3}$ & $\mathrm{~N}_{0} \mathrm{~A}_{1} \mathrm{~V}_{3}$ & $N_{1} A_{0} V_{3}$ & $N_{1} A_{1} V_{3}$ & $\mathrm{~N}_{0} \mathrm{~A}_{0} \mathrm{~V}_{4}$ & $\mathrm{~N}_{0} \mathrm{~A}_{1} \mathrm{~V}_{4}$ & $\mathrm{~N}_{1} \mathrm{~A}_{0} \mathrm{~V}_{4}$ & $N_{1} A_{1} V_{4}$ \\
\hline \multicolumn{9}{|c|}{$\begin{array}{l}{ }^{6} \text { Contained: Phosphatidyl-choline (PC) 16\%, Phosphatidyl-ethanolamine (PE) 10\%, Phosphatidic-acid (PA) } 11 \% \text {, Phosphatidyl- } \\
\text { inositol (PI) 8\%, Lyso-phosphatidyl-choline (LPC) 8\%, Lyso-phosphatidyl-ethanolamine (LPE) } 13 \% \text {, Lyso-phosphatidic-acid } \\
\text { (LPA) 6\%, Lyso-phosphatidyl-inositol (LPI) 8\%, Carrier 20\% (Brand-Molimen, Country of origin-Spain); }\end{array}$} \\
\hline \multicolumn{9}{|c|}{${ }^{7}$ Unit was considered as $\%$ or otherwise stated; } \\
\hline \multicolumn{9}{|c|}{${ }^{8}$ As per standard procedure (AOAC, 2019); } \\
\hline
\end{tabular}

\section{Chemical analysis}

Chemical analyses of the experimental diets were carried out in triplicate for dry matter (DM), crude protein (CP), crude fiber (CF), nitrogen free extracts (NFE), ether extracts (EE) and total ash (TA) in the animal nutrition postgraduate laboratory, Chattogram Veterinary and Animal Sciences University, Chattogram as per standard procedure (AOAC, 2019). Moisture was estimated by Hot air oven (SLN-115, Pol-Eko-Aparatus SP.J, Poland). CP was estimated by micro Kjeldhal apparatus (Kjeldhal digestion unit SBS800, Kjeldhal distillation unit D1000, FoodAlyt, Germany). CF was estimated by using Ankom Fiber Analyzer (Fiberbag System-6, Gerhardt, Germany). EE was estimated by using Soxtec (RS-232, SER-148, VelpScientifica, 155 Keyland Court, Bohemia, NY 11716 - US). TA was estimated by the muffle furnace (HYSC, Non Yong Scientific Equipment Company Ltd., 874-1 Wolgye 4-dong, Nowon-gu, Seoul, Korea). Apparent gross energy (AGE) of mixed diets was estimated by using the bomb calorimeter (Parr 6200 Calorimeter, Parr Instruments Co., USA).

\section{Performance parameter}

Mortality was recorded as occurred, while average daily feed intake (ADFI), average daily gain (ADG), feed efficiency (FE) were recorded fortnightly. Carcass characteristics, hematological and biochemical parameters were recorded at 5th week. Weight gain was calculated by deducting initial body weight from the final body weight of the birds. Feed intake was calculated by deducting leftover from the total feeds supplied to the birds. The FE was calculated dividing feed intake by weight gain.

\section{Carcass characteristics}

At day 35 two birds from each replicate were randomly selected and killed by severing the jugular vein and carotid artery. Once a bird was adequately bleed out, it was scalded and defeathered. After defeathering, the birds were eviscerated and the head and feet were removed as per standard technique (Jones, 1984). During evisceration process, abdominal fat, lung, liver, kidney, spleen, gizzard and proventriculus were excised separately and weighed. Dressed birds were weighed to obtain a dressed carcass weight.

\section{Cardio-pulmonary morphometry}

The heart was isolated from the carcass immediate after slaughter. The data of heart weight, right ventricular weight, left ventricular weight, right ventricular diameter, left ventricular diameter, right ventricular thickness and left ventricular thickness were measured thereafter by using slide caliper (WiikaVernier Caliper, $150 \mathrm{~mm}$, WA-VC1150) and digital screw gauge (Mitutoyo, Quickmini, Mitutoyo corporation, Japan). The diameter of left and right ventricles were measured perpendicular to the long axis, at the maximum measureable dimensions and mean values were calculated.

\section{Hemato-biochemical test}

Samples were analyzed in three different research laboratories, i.e., Postgraduate laboratory of Dairy and Poultry Science, Animal science and Nutrition laboratory and Physiology, Pharmacology and Biochemistry laboratory of CVASU. Blood samples were collected from the carotid artery of three birds from each replicate using $4 \mathrm{ml}$ sterile vacutainer tubes containing anticoagulant (EDTA) for serum tests and without anticoagulant for hematology. The hemoglobin concentration was estimated by hematology analyzer (Celltac Alpha, Nihon Kohden, MEK 6550). Packed cell volume was measured after centrifugation of a small amount of 
blood using micro-hematocrit capillary tubes. For serum analysis, clotted blood in the vacutainer tube was centrifuged at 3000 rpm for 20 minutes by the multipurpose centrifuge (DLAB, DM0636, Berkeley, CA $94720-3030$ ) and prepared serum was collected into the eppendorf tube by micropipette. Sera samples were marked and stored in $-20^{\circ} \mathrm{C}$ until being analyzed. All biochemical tests were conducted by a hemato-biochemical analyzer (HumaLyzer 3000, Human, Germany). Manufacturer's recommended standard test kits were used for testing serum glucose (Method: GOD-PAP, Ref. 10260, Liquicolor, Human, Germany); serum glutamic oxaloacetic transaminase, SGOT (Method: ALAT IFCC, Ref. 12021, Human, Germany), serum glutamate-pyruvate transaminase, SGPT (Method: ALAT IFCC, Ref. 12022, Human, Germany), albumin (Ref. 10560, Human, Germany); total protein (Ref. 10570, Human, Germany), total cholesterol (Ref. CH 200, Randox, Germany) and $\mathrm{T}_{3}$ (Bioscience medical, Madrid, Spain).

\section{Statistical analysis}

Data were compiled in MS Excel. Raw data related to weight gain, feed intake, FCR, carcass characteristics, cardio-pulmonary morphometry, hematological and biochemical parameters were tested for outliers and multicollinearity by inter quartile range test and variance inflation factors. Normality of variable was checked by using normal probability plot and equality of variances in the response variable was checked by Shapiro Wilk test. Data were analyzed in generalized linear model (GLM) by using Stata 14.1 SE (StataCorp LP, College Station, Texas, USA). Means showing significant differences were compared by Duncan's New Multiple Range Test (Duncan, 1955). Statistical significance was accepted at $p<0.05$ for Fisher's F-tests. The following statistical model was used:

\begin{tabular}{|c|c|c|}
\hline Yijk & $=$ & $\mu+a i+\beta j+\gamma k+(a \beta \gamma) i j k+\varepsilon i j k n$ \\
\hline \multicolumn{3}{|l|}{ Where, } \\
\hline$\mu$ & $=$ & The intercept of the regression model; \\
\hline ai & $=$ & $\begin{array}{l}\text { The effect of the 'ith' level of the factor ' } a \text { ' }\left(\mathrm{NaHCO}_{3}\right) \text { on the value observed in } \mathrm{Yijk}\left(\mathrm{i}=0,0.10 \% \text { of } \mathrm{NaHCO}_{3} \text { of }\right. \\
\text { the basal diet); }\end{array}$ \\
\hline$\beta \mathrm{j}$ & $=$ & $\begin{array}{l}\text { The effect of the ' } \mathrm{j} \text { th' level of the factor ' } \beta \text { ' (Additive) on the value observed in Yijk }(j=0,0.10 \% \text { of } L \text {-arginine }+ \\
\text { vitamin-C of the basal diet); }\end{array}$ \\
\hline yk & $=$ & $\begin{array}{l}\text { The effect of the ' } k \text { th' level of the factor ' } Y \text { ' (Vegetable oil) on the value observed in Yijk }(k=3,4 \% \text { of vegetable } \\
\text { oil of the basal diet); }\end{array}$ \\
\hline$(a \beta \gamma) i j k$ & $=$ & $\begin{array}{l}\text { The interaction effect of the of the ' } i \text { th' level of the factor ' } a \text { ', the ' } j{ }^{\text {th' }} \text { level of the factor ' } \beta \text { ', and ' } k \text { th' level of the } \\
\text { factor ' }{ }^{\prime} \text { '; }\end{array}$ \\
\hline Yijk & $=$ & $\begin{array}{l}\text { The observed value of the variable under study for the ' } n{ }^{\text {th' }} \text { repetition of the combination of the ' } \mathrm{i}^{\text {th' }} \text { level of } \\
\text { factor ' } a \text { ', the ' } j \text { th' level of the factor ' } \beta \text { ', and the ' } k \text { th' level of the factor ' } \gamma \text { '; }\end{array}$ \\
\hline$\varepsilon i j k$ & $=$ & $\begin{array}{l}\text { The random sampling error due to ' } i \text { 'th' level of the factor ' } a \text { ', ' } j \text { th' level of the factor ' } \beta \text { ', and ' } k \text { th' level of the } \\
\text { factor ' } y \text { '. }\end{array}$ \\
\hline
\end{tabular}

\section{Results}

\section{Performance}

Supplementation of $\mathrm{NaHCO}_{3}$ decreased $(p<0.001)$ ADFI at 1-14 d, 15-35 d, 1-35 d and improved $(p<0.01)$ FE from 0.91 to 0.86 at $1-14 d$, although, $A D G$ remained unchanged $(p>0.05)$. Dietary L-arginine + vitamin-C did not influence $(p>0.05)$ the performance parameter of the birds throughout the trial period. Vegetable oil decreased $(p<0.01)$ ADFI at 1-14d, 15-35 $d, 1-35$ $d$, increased $(p<0.01)$ ADG at 1-14d and improved $(p<0.001)$ FE at 1-14 d. There were no interactions of $\mathrm{NaHCO}_{3} \times \mathrm{L}-$ arginine + vitamin- $\mathrm{C} \times$ Vegetable oil on performance parameters of the experimental birds (Table 4). 
Table 4

Initial live weight (ILW, g/bird/d), final live weight (FLW, g/bird/d), average daily feed intake (ADFI, g/bird/d), average daily gain $(A D G, g / b i r d / d)$ and feed efficiency (FE, ADFl/ADG) of the broiler birds fed diets supplemented with various levels of $\mathrm{NaHCO}_{3}, \mathrm{~L}-$ arginine + vitamin-C and vegetable oils

\begin{tabular}{|c|c|c|c|c|c|c|c|c|c|c|c|}
\hline \multirow{2}{*}{$\begin{array}{l}\text { Treatment } \\
\text { factors }^{1}\end{array}$} & \multicolumn{11}{|c|}{ Performance of broiler birds } \\
\hline & \multirow{2}{*}{$\begin{array}{l}\text { ILW } \\
\text { (g/bird/d) } \\
15-35 d\end{array}$} & \multirow{2}{*}{$\begin{array}{l}\text { FLW } \\
\text { (g/bird/d) } \\
1-35 d\end{array}$} & \multicolumn{3}{|c|}{ ADFI (g/bird/d) } & \multicolumn{3}{|c|}{ ADG (g/bird/d) } & \multicolumn{3}{|l|}{ FE } \\
\hline $1-14 d$ & & & $\begin{array}{l}1-14 \\
d\end{array}$ & $\begin{array}{l}15-35 \\
d\end{array}$ & $\begin{array}{l}1-35 \\
d\end{array}$ & $\begin{array}{l}1-14 \\
d\end{array}$ & $\begin{array}{l}15- \\
35 d\end{array}$ & $\begin{array}{l}1-35 \\
d\end{array}$ & & & \\
\hline \multicolumn{12}{|c|}{$\mathrm{NaHCO} 3(\mathrm{~N})$} \\
\hline $0.00 \%$ & 40.97 & 2109.91 & $18.54^{\mathrm{a}}$ & $115.08^{a}$ & $76.47^{a}$ & 20.36 & 74.08 & 52.59 & $0.91^{\mathrm{a}}$ & 1.54 & 1.29 \\
\hline $0.10 \%$ & 42.73 & 2187.86 & $17.56^{\mathrm{b}}$ & $113.75^{b}$ & $75.28^{b}$ & 20.33 & 73.25 & 52.08 & $0.86^{b}$ & 1.55 & 1.27 \\
\hline \multicolumn{12}{|c|}{ L-arginine + vitamin-C (A) } \\
\hline $0.00 \%$ & 41.48 & 2123.89 & 18.00 & 114.33 & 75.80 & 20.48 & 72.52 & 51.71 & 0.88 & 1.56 & 1.29 \\
\hline $0.10 \%$ & 42.22 & 2173.87 & 18.10 & 114.50 & 75.94 & 20.21 & 74.81 & 52.97 & 0.89 & 1.53 & 1.27 \\
\hline \multicolumn{12}{|c|}{$\begin{array}{l}\text { Vegetable oil } \\
\text { (V) }\end{array}$} \\
\hline $3.00 \%$ & 42.13 & 2120.50 & $18.48^{a}$ & $115.00^{\mathrm{a}}$ & $76.39^{a}$ & $19.93^{\mathrm{a}}$ & 74.75 & 52.82 & $0.92^{\mathrm{a}}$ & 1.54 & 1.29 \\
\hline $4.00 \%$ & 41.57 & 2177.26 & $17.63^{\mathrm{b}}$ & $113.83^{b}$ & $75.35^{\mathrm{b}}$ & $20.76^{b}$ & 72.58 & 51.85 & $0.85^{b}$ & 1.55 & 1.27 \\
\hline \multicolumn{12}{|l|}{$N \times A \times V$} \\
\hline $\mathrm{N}_{0} \times \mathrm{A}_{0} \times \mathrm{V}_{3}$ & 41.00 & 2048.91 & 18.75 & 115.33 & 76.70 & 20.02 & 74.54 & 52.73 & 0.93 & 1.54 & 1.30 \\
\hline $\mathrm{N}_{0} \times \mathrm{A}_{1} \times \mathrm{V}_{3}$ & 40.73 & 2129.29 & 18.00 & 114.33 & 75.80 & 20.88 & 72.23 & 51.69 & 0.86 & 1.55 & 1.28 \\
\hline $\mathrm{N}_{0} \times \mathrm{A}_{0} \times \mathrm{V}_{4}$ & 40.00 & 2104.76 & 19.42 & 116.33 & 77.57 & 20.19 & 75.88 & 53.61 & 0.96 & 1.52 & 1.30 \\
\hline $\mathrm{N}_{0} \times \mathrm{A}_{1} \times \mathrm{V}_{4}$ & 42.13 & 2156.67 & 18.00 & 114.33 & 75.80 & 20.35 & 73.67 & 52.34 & 0.88 & 1.53 & 1.27 \\
\hline $\mathrm{N}_{1} \times \mathrm{A}_{0} \times \mathrm{V}_{3}$ & 42.75 & 2160.71 & 18.00 & 114.33 & 75.80 & 20.05 & 72.66 & 51.62 & 0.89 & 1.57 & 1.30 \\
\hline
\end{tabular}

${ }^{1} \mathrm{~N}_{0} \mathrm{~A}_{1} \mathrm{~V}_{3}=\mathrm{NaHCO}_{3} \mathrm{O} \%$, L-arginine $0.05 \%+$ Vitamin-C $0.05 \%$, Vegetable oil $3 \%$;

$\mathrm{N}_{1} \mathrm{~A}_{0} \mathrm{~V}_{3}=\mathrm{NaHCO}_{3} 0.1 \%$, L-arginine $0 \%+$ Vitamin-C $0 \%$, vegetable oil $3 \%$;

$\mathrm{N}_{1} \mathrm{~A}_{1} \mathrm{~V}_{3}=\mathrm{NaHCO}_{3} 0.1 \%$, L-arginine $0.05 \%+$ Vitamin-C $0.05 \%$, Vegetable oil 3\%;

$\mathrm{N}_{0} \mathrm{~A}_{0} \mathrm{~V}_{4}=\mathrm{NaHCO}_{3} 0 \%$, L-arginine $0 \%+$ Vitamin-C $0 \%$, Vegetable oil $4 \% ;$

$\mathrm{N}_{0} \mathrm{~A}_{1} \mathrm{~V}_{4}=\mathrm{NaHCO}_{3} \mathrm{0} \%$, L-arginine $0.05 \%+$ Vitamin-C $0.05 \%$, Vegetable oil $4 \%$;

$\mathrm{N}_{1} \mathrm{~A}_{0} \mathrm{~V}_{4}=\mathrm{NaHCO}_{3} 0.1 \%$, L-arginine $0 \%+$ Vitamin-C $0 \%$, Vegetable oil $4 \%$;

$\mathrm{N}_{1} \mathrm{~A}_{1} \mathrm{~V}_{4}=\mathrm{NaHCO}_{3} 0.1 \%$, L-arginine $0.05 \%+$ Vitamin-C $0.05 \%$, Vegetable oil $4 \%$;

${ }^{2} \mathrm{SEM}=$ Standard error of the means;

${ }^{3}$ NS $=$ Non-significant $(p>0.05), *=$ Significant $(p<0.05),{ }^{* *}=$ Significant $(p<0.01), * \star \star=$ Significant $(p<0.001)$;

${ }^{a-b}$ Means bearing different superscripts in a column differ $(p<0.05)$. 


\begin{tabular}{|c|c|c|c|c|c|c|c|c|c|c|c|}
\hline \multirow{2}{*}{$\begin{array}{l}\text { Treatment } \\
\text { factors }{ }^{1}\end{array}$} & \multicolumn{11}{|c|}{ Performance of broiler birds } \\
\hline & \multirow{3}{*}{$\begin{array}{l}\text { ILW } \\
\text { (g/bird/d) } \\
41.43\end{array}$} & \multirow{3}{*}{$\begin{array}{l}\text { FLW } \\
\text { (g/bird/d) } \\
2156.67\end{array}$} & \multicolumn{3}{|c|}{ ADFI (g/bird/d) } & \multicolumn{3}{|c|}{ ADG (g/bird/d) } & \multicolumn{3}{|l|}{ FE } \\
\hline & & & & & & & & & & & \\
\hline $\mathrm{N}_{1} \times \mathrm{A}_{1} \times \mathrm{V}_{3}$ & & & 17.25 & 113.33 & 74.90 & 20.95 & 70.66 & 50.78 & 0.82 & 1.58 & 1.28 \\
\hline $\mathrm{N}_{1} \times \mathrm{A}_{0} \times \mathrm{V}_{4}$ & 44.75 & 2167.62 & 17.75 & 114.00 & 75.50 & 19.45 & 75.93 & 53.34 & 0.91 & 1.53 & 1.28 \\
\hline $\mathrm{N}_{1} \times \mathrm{A}_{1} \times \mathrm{V}_{4}$ & 42.00 & 2266.43 & 17.25 & 113.33 & 74.90 & 20.86 & 73.75 & 52.59 & 0.82 & 1.52 & 1.24 \\
\hline SEM $^{12}$ & 0.28 & 14.65 & 0.19 & 0.25 & 0.23 & 0.16 & 0.69 & 0.41 & 0.01 & 0.01 & 0.01 \\
\hline \multicolumn{12}{|l|}{ Significance ${ }^{13}$} \\
\hline $\mathrm{NaHCO}_{3}$ & NS & NS & $\star * \star$ & $\star * *$ & $\star \star \star *$ & NS & NS & NS & ** & NS & NS \\
\hline $\begin{array}{l}\text { L-arg. }+ \\
\text { vitamin-C }\end{array}$ & NS & $\star \star$ & NS & NS & NS & NS & NS & NS & NS & NS & NS \\
\hline Vegetable oil & NS & * & ** & ** & ** & ** & NS & NS & $\star \star \star ~$ & NS & NS \\
\hline $\begin{array}{l}\mathrm{NaHCO} \times \mathrm{L}- \\
\text { arg.×Veg. }\end{array}$ & NS & NS & NS & NS & NS & NS & NS & NS & NS & NS & NS \\
\hline \multicolumn{12}{|c|}{${ }^{1} \mathrm{~N}_{0} \mathrm{~A}_{1} \mathrm{~V}_{3}=\mathrm{NaHCO}_{3} \mathrm{O} \%$, L-arginine $0.05 \%+$ Vitamin-C $0.05 \%$, Vegetable oil $3 \%$; } \\
\hline \multicolumn{12}{|c|}{$\mathrm{N}_{1} \mathrm{~A}_{0} \mathrm{~V}_{3}=\mathrm{NaHCO}_{3} 0.1 \%$, L-arginine $0 \%+$ Vitamin-C $0 \%$, vegetable oil $3 \%$; } \\
\hline \multicolumn{12}{|c|}{$\mathrm{N}_{1} \mathrm{~A}_{1} \mathrm{~V}_{3}=\mathrm{NaHCO}_{3} 0.1 \%$, L-arginine $0.05 \%+$ Vitamin-C $0.05 \%$, Vegetable oil $3 \%$; } \\
\hline \multicolumn{12}{|c|}{$\mathrm{N}_{0} \mathrm{~A}_{0} \mathrm{~V}_{4}=\mathrm{NaHCO}_{3} 0 \%, \mathrm{~L}$-arginine $0 \%+$ Vitamin-C $0 \%$, Vegetable oil $4 \%$} \\
\hline \multicolumn{12}{|c|}{$\mathrm{N}_{0} \mathrm{~A}_{1} \mathrm{~V}_{4}=\mathrm{NaHCO}_{3} \mathrm{O} \%$, L-arginine $0.05 \%+$ Vitamin-C $0.05 \%$, Vegetable oil $4 \%$} \\
\hline \multicolumn{12}{|c|}{$\mathrm{N}_{1} \mathrm{~A}_{0} \mathrm{~V}_{4}=\mathrm{NaHCO}_{3} 0.1 \%$, L-arginine $0 \%+$ Vitamin-C $0 \%$, Vegetable oil $4 \%$} \\
\hline \multicolumn{12}{|c|}{$\mathrm{N}_{1} \mathrm{~A}_{1} \mathrm{~V}_{4}=\mathrm{NaHCO}_{3} 0.1 \%$, L-arginine $0.05 \%+$ Vitamin-C $0.05 \%$, Vegetable oil $4 \%$; } \\
\hline \multicolumn{12}{|c|}{${ }^{2} \mathrm{SEM}=$ Standard error of the means; } \\
\hline \multicolumn{12}{|c|}{${ }^{3} N S=$ Non-significant $(p>0.05), *=$ Significant $(p<0.05), * *=$ Significant $(p<0.01), * \star *=$ Significant $(p<0.001)$} \\
\hline
\end{tabular}

\section{Carcass characteristics}

Main as well as interaction effects of supplementing $\mathrm{NaHCO}_{3}$, L-arginine + vitamin-C and vegetable oil appeared nil $(p>0.05)$ on the carcass characteristics of the experimental broiler birds (Table 5). 
Table 5

Carcass characteristics of the broiler birds fed diets supplemented with various levels of $\mathrm{NaHCO}_{3}$ L-arginine + vitamin-C and vegetable oils

\section{Treatment factors $^{1} \quad$ Relative weight of the carcass components as \% live weight}

$\begin{array}{llllllllllll}\mathrm{DP}^{2} & \mathrm{BRW}^{3} & \mathrm{BKW}^{4} & \mathrm{TW}^{5} & \mathrm{DW}^{6} & \mathrm{SW}^{7} & \mathrm{WW}^{8} & \mathrm{NW}^{9} & \mathrm{LW}^{10} & \mathrm{GW}^{11} & \mathrm{PW}^{12} & \mathrm{AFW}^{13}\end{array}$

$\operatorname{NaHCO} 3(\mathrm{~N})$

$\begin{array}{lllllllllllll}0.00 \% & 62.05 & 16.72 & 11.00 & 7.58 & 6.99 & 3.57 & 4.22 & 2.14 & 1.85 & 2.58 & 0.45 & 1.31 \\ 0.10 \% & 60.04 & 16.40 & 10.60 & 7.40 & 6.65 & 3.64 & 4.11 & 1.95 & 2.10 & 2.45 & 0.39 & 1.16\end{array}$

L-arginine + vitamin-C (A)

\begin{tabular}{lllllllllllll}
$0.00 \%$ & 61.39 & 16.68 & 10.72 & 7.61 & 6.90 & 3.62 & 4.19 & 2.08 & 2.06 & 2.49 & 0.41 & 1.15 \\
\hline $0.10 \%$ & 60.70 & 16.44 & 10.88 & 7.38 & 6.73 & 3.58 & 4.14 & 2.01 & 1.89 & 2.53 & 0.43 & 1.32
\end{tabular}

Vegetable oil (V)

\begin{tabular}{|llllllllllllll}
$3.00 \%$ & 61.36 & 16.81 & 10.67 & 7.57 & 6.87 & 3.88 & 4.23 & 1.96 & 1.88 & 2.54 & 0.42 & 1.06 \\
\hline $4.00 \%$ & 60.73 & 16.32 & 10.93 & 7.41 & 6.77 & 3.33 & 4.10 & 2.12 & 2.08 & 2.49 & 0.42 & 1.41 \\
\hline $\mathrm{N} \times \mathrm{A} \times \mathrm{V}$ & & & & & & & & & & & & \\
\hline $\mathrm{N}_{0} \times \mathrm{A}_{0} \times \mathrm{V}_{3}$ & 63.22 & 17.57 & 10.76 & 7.71 & 7.21 & 3.92 & 4.34 & 2.08 & 1.98 & 2.51 & 0.46 & 0.98 \\
\hline $\mathrm{N}_{0} \times \mathrm{A}_{1} \times \mathrm{V}_{3}$ & 61.89 & 16.81 & 11.31 & 7.59 & 6.64 & 3.15 & 3.90 & 2.16 & 1.85 & 2.51 & 0.38 & 1.55 \\
\hline $\mathrm{N}_{0} \times \mathrm{A}_{0} \times \mathrm{V}_{4}$ & 61.59 & 16.38 & 11.18 & 7.65 & 6.87 & 3.75 & 4.04 & 2.01 & 1.73 & 2.72 & 0.51 & 1.23 \\
\hline $\mathrm{N}_{0} \times \mathrm{A}_{1} \times \mathrm{V}_{4}$ & 61.51 & 16.14 & 10.74 & 7.39 & 7.23 & 3.44 & 4.60 & 2.30 & 1.85 & 2.58 & 0.45 & 1.49 \\
\hline $\mathrm{N}_{1} \times \mathrm{A}_{0} \times \mathrm{V}_{3}$ & 60.15 & 16.25 & 10.40 & 7.43 & 6.87 & 4.07 & 4.46 & 2.07 & 1.96 & 2.58 & 0.35 & 0.86 \\
\hline $\mathrm{N}_{1} \times \mathrm{A}_{1} \times \mathrm{V}_{3}$ & 60.31 & 16.11 & 10.42 & 7.70 & 6.90 & 3.35 & 4.06 & 2.00 & 2.46 & 2.38 & 0.43 & 1.21 \\
\hline $\mathrm{N}_{1} \times \mathrm{A}_{0} \times \mathrm{V}_{4}$ & 60.49 & 17.03 & 10.34 & 7.50 & 6.52 & 3.75 & 4.08 & 1.70 & 1.84 & 2.34 & 0.35 & 1.17 \\
\hline $\mathrm{N}_{1} \times \mathrm{A}_{1} \times \mathrm{V}_{4}$ & 59.22 & 16.21 & 11.25 & 6.98 & 6.30 & 3.38 & 3.83 & 2.02 & 2.15 & 2.48 & 0.41 & 1.40 \\
\hline $\mathrm{SEM}^{12}$ & 0.28 & 14.65 & 0.58 & 0.29 & 0.18 & 0.12 & 0.13 & 0.08 & 0.08 & 0.07 & 0.08 & 0.06 \\
\hline
\end{tabular}

Significance ${ }^{13}$

\begin{tabular}{|lllllllllllll|}
$\mathrm{NaHCO}_{3}$ & NS & NS & NS & NS & NS & NS & NS & NS & NS & NS & NS & NS \\
\hline L-arg. + vitamin-C & NS & NS & NS & NS & NS & NS & NS & NS & NS & NS & NS & NS \\
\hline $\begin{array}{l}\text { Vegetable oil } \\
\text { NS }\end{array}$ & NS & NS & NS & NS & NS & NS & NS & NS & NS & NS & NS \\
$\begin{array}{l}\text { NaHCO3 } \times \text { L- } \\
\text { arg. } \times \text { Veg. }\end{array}$ & NS & NS & NS & NS & NS & NS & NS & NS & NS & NS & NS & NS \\
\hline
\end{tabular}

${ }^{1} \mathrm{~N}_{0} \mathrm{~A}_{1} \mathrm{~V}_{3}=\mathrm{NaHCO}_{3} \mathrm{0} \%$, L-arginine $0.05 \%+$ Vitamin-C 0.05\%, Vegetable oil 3\%;

$\mathrm{N}_{1} \mathrm{~A}_{0} \mathrm{~V}_{3}=\mathrm{NaHCO}_{3} 0.1 \%$, L-arginine $0 \%+$ Vitamin-C $0 \%$, vegetable oil $3 \%$;

$\mathrm{N}_{1} \mathrm{~A}_{1} \mathrm{~V}_{3}=\mathrm{NaHCO}_{3}$ 0.1\%, L-arginine $0.05 \%$ + Vitamin-C 0.05\%, Vegetable oil 3\%;

$\mathrm{N}_{0} \mathrm{~A}_{0} \mathrm{~V}_{4}=\mathrm{NaHCO}_{3} 0 \%$, L-arginine $0 \%+$ Vitamin-C $0 \%$, Vegetable oil $4 \%$; 


\begin{tabular}{|c|c|c|c|c|c|c|c|c|c|c|c|c|}
\hline \multirow[t]{2}{*}{ Treatment factors ${ }^{1}$} & \multicolumn{12}{|c|}{ Relative weight of the carcass components as $\%$ live weight } \\
\hline & $\mathrm{DP}^{2}$ & BRW $^{3}$ & $\mathrm{BKW}^{4}$ & $\mathrm{TW}^{5}$ & $\mathrm{DW}^{6}$ & $s w^{7}$ & $w^{8} w^{8}$ & $\mathrm{NW}^{9}$ & $\mathrm{LW}^{10}$ & $\mathrm{GW}^{11}$ & PW 12 & AFW ${ }^{13}$ \\
\hline \multicolumn{13}{|c|}{$\mathrm{N}_{0} \mathrm{~A}_{1} \mathrm{~V}_{4}=\mathrm{NaHCO}_{3} \mathrm{0} \%$, L-arginine $0.05 \%+$ Vitamin-C $0.05 \%$, Vegetable oil $4 \%$} \\
\hline \multicolumn{13}{|c|}{$\mathrm{N}_{1} \mathrm{~A}_{0} \mathrm{~V}_{4}=\mathrm{NaHCO}_{3} 0.1 \%$, L-arginine $0 \%+$ Vitamin-C $0 \%$, Vegetable oil $4 \%$} \\
\hline \multicolumn{13}{|c|}{$\mathrm{N}_{1} \mathrm{~A}_{1} \mathrm{~V}_{4}=\mathrm{NaHCO}_{3} 0.1 \%$, L-arginine $0.05 \%+$ Vitamin-C $0.05 \%$, Vegetable oil $4 \%$; } \\
\hline \multicolumn{13}{|c|}{$\begin{array}{l}{ }^{2} \mathrm{DP}=\text { Dressing percentage, }{ }^{3} \mathrm{BRW}=\text { Breast weight, }{ }^{4} \mathrm{BKW}=\mathrm{B} \text { ack weight, }{ }^{5} \mathrm{TW}=\text { Thigh weight, }{ }^{6} \mathrm{DW}=\mathrm{Drumstick} \text { weight, }{ }^{7} \mathrm{SW}=\mathrm{Shank} \\
\text { weight, }{ }^{8} \mathrm{WW}=\text { Wing weight, }{ }^{9} \mathrm{NW}=\text { Neck weight, }{ }^{10} \mathrm{LW}=\mathrm{Liver} \text { weight, }{ }^{11} \mathrm{GW}=\text { Gizzard weight, }{ }^{12} \mathrm{PW}=\text { Proventriculus weight, } \\
{ }^{13} \mathrm{AFW}=\mathrm{Abdominal} \text { fat weight; }\end{array}$} \\
\hline \multicolumn{13}{|c|}{${ }^{14}$ SEM = Standard error of the means; } \\
\hline
\end{tabular}

\section{Cardio-pulmonary morphometry}

Supplementation of L-arginine + vitamin-C decreased $(p<0.01)$ the heart rate. Further, $\mathrm{NaHCO}_{3} \times \mathrm{L}$-arginine + vitamin- $\mathrm{C} \times$ Vegetable oil additively interacted to decrease $(p<0.01)$ the weight of right and left ventricles in terms of heart weight, the ratio of $\mathrm{RV}$ :TV and heart rate but did not exceed the normal thresholds (Table 6). 
Table 6

Cardio-pulmonary morphometry of the broiler birds fed diets supplemented with various levels of $\mathrm{NaHCO}_{3}$, L-arginine + vitamin-C and vegetable oils

\begin{tabular}{|c|c|c|c|c|c|c|c|c|c|c|}
\hline \multirow[t]{2}{*}{ Treatment factors ${ }^{1}$} & \multicolumn{10}{|c|}{ Cardio-pulmonary morphometry } \\
\hline & $\mathrm{HW}^{2}$ & $\mathrm{LW}^{3}$ & $\mathrm{RVW}^{4}$ & LVW $^{5}$ & $\mathrm{RV} / \mathrm{HW}^{6}$ & $\mathrm{LV} / \mathrm{HW}^{7}$ & $\mathrm{RV} / \mathrm{TV}^{8}$ & $\mathrm{RVT}^{9}$ & RVD $^{10}$ & $\mathrm{HB}^{11}$ \\
\hline \multicolumn{11}{|l|}{$\mathrm{NaHCO} 3(\mathrm{~N})$} \\
\hline $0.00 \%$ & 14.13 & 12.00 & 0.66 & 3.13 & 5.05 & 22.12 & 0.19 & 1.63 & 5.88 & 387.78 \\
\hline $0.10 \%$ & 13.13 & 11.75 & 0.66 & 2.88 & 4.69 & 21.90 & 0.17 & 1.50 & 5.00 & 384.78 \\
\hline \multicolumn{11}{|c|}{ L-arginine + vitamin-C (A) } \\
\hline $0.00 \%$ & 13.50 & 13.63 & 0.68 & 2.88 & 5.00 & 21.82 & 0.19 & 1.63 & 5.75 & $390.00^{2}$ \\
\hline $0.10 \%$ & 13.75 & 11.25 & 0.66 & 3.00 & 4.82 & 21.30 & 0.18 & 1.63 & 5.25 & $382.50^{b}$ \\
\hline \multicolumn{11}{|l|}{ Vegetable oil (V) } \\
\hline $3.00 \%$ & 13.38 & 12.25 & 0.63 & 2.88 & 4.67 & 21.50 & 0.17 & 1.63 & 5.38 & 383.28 \\
\hline $4.00 \%$ & 13.88 & 11.50 & 0.63 & 3.00 & 4.50 & 21.62 & 0.18 & 1.50 & 5.63 & 388.50 \\
\hline \multicolumn{11}{|l|}{$\mathrm{N} \times \mathrm{A} \times \mathrm{V}$} \\
\hline $\mathrm{N}_{0} \times \mathrm{A}_{0} \times \mathrm{V}_{3}$ & 15.00 & 13.38 & 0.76 & 3.25 & 5.08 & 21.67 & 0.19 & 1.75 & 5.88 & 395.00 \\
\hline $\mathrm{N}_{0} \times \mathrm{A}_{1} \times \mathrm{V}_{3}$ & 13.75 & 12.50 & 0.58 & 3.13 & 4.18 & 22.73 & 0.16 & 1.50 & 5.88 & 385.00 \\
\hline $\mathrm{N}_{0} \times \mathrm{A}_{0} \times \mathrm{V}_{4}$ & 13.75 & 11.25 & 0.59 & 3.13 & 4.27 & 22.73 & 0.16 & 1.25 & 6.25 & 397.50 \\
\hline $\mathrm{N}_{0} \times \mathrm{A}_{1} \times \mathrm{V}_{4}$ & 13.75 & 10.88 & 0.79 & 2.88 & 5.73 & 20.91 & 0.22 & 2.13 & 5.63 & 372.50 \\
\hline $\mathrm{N}_{1} \times \mathrm{A}_{0} \times \mathrm{V}_{3}$ & 10.88 & 12.88 & 0.65 & 2.25 & 5.98 & 20.69 & 0.22 & 1.63 & 5.00 & 390.00 \\
\hline $\mathrm{N}_{1} \times \mathrm{A}_{1} \times \mathrm{V}_{3}$ & 13.75 & 10.38 & 0.70 & 3.00 & 5.09 & 21.82 & 0.16 & 1.50 & 4.75 & 362.50 \\
\hline $\mathrm{N}_{1} \times \mathrm{A}_{0} \times \mathrm{V}_{4}$ & 14.13 & 12.50 & 0.68 & 3.13 & 4.78 & 22.12 & 0.18 & 1.63 & 5.88 & 375.00 \\
\hline $\mathrm{N}_{1} \times \mathrm{A}_{1} \times \mathrm{V}_{4}$ & 13.75 & 11.25 & 0.60 & 3.13 & 4.36 & 22.73 & 0.19 & 1.25 & 4.63 & 410.00 \\
\hline SEM $^{12}$ & 0.48 & 0.43 & 0.03 & 0.11 & 0.23 & 0.29 & 0.01 & 0.10 & 0.23 & 5.89 \\
\hline \multicolumn{11}{|l|}{ Significance $e^{13}$} \\
\hline $\mathrm{NaHCO}_{3}$ & NS & NS & NS & NS & NS & NS & NS & NS & NS & NS \\
\hline L-arg. + vitamin-C & NS & NS & NS & NS & NS & NS & NS & NS & NS & $\star *$ \\
\hline Vegetable oil & NS & NS & NS & NS & NS & NS & NS & NS & NS & NS \\
\hline $\mathrm{NaHCO} \times$ L-arg. $\times$ Veg. & NS & NS & NS & NS & ** & ** & ** & NS & NS & $\star *$ \\
\hline \multicolumn{11}{|c|}{${ }^{1} \mathrm{~N}_{0} \mathrm{~A}_{1} \mathrm{~V}_{3}=\mathrm{NaHCO}_{3} 0 \%$, L-arginine $0.05 \%+$ Vitamin-C $0.05 \%$, Vegetable oil $3 \%$; } \\
\hline \multicolumn{11}{|c|}{$\mathrm{N}_{1} \mathrm{~A}_{0} \mathrm{~V}_{3}=\mathrm{NaHCO}_{3} 0.1 \%$, L-arginine $0 \%+$ Vitamin-C $0 \%$, vegetable oil $3 \%$; } \\
\hline \multicolumn{11}{|c|}{$\mathrm{N}_{1} \mathrm{~A}_{1} \mathrm{~V}_{3}=\mathrm{NaHCO}_{3} 0.1 \%$, L-arginine $0.05 \%+$ Vitamin-C $0.05 \%$, Vegetable oil $3 \%$; } \\
\hline $\mathrm{N}_{0} \mathrm{~A}_{0} \mathrm{~V}_{4}=\mathrm{NaHCO}_{3} 0 \%$ & rginin & $\%+\mathrm{Vit}$ & in- 0 & Vegeta & oil $4 \%$ & & & & & \\
\hline
\end{tabular}




\begin{tabular}{|c|c|c|c|c|c|c|c|c|c|c|}
\hline \multirow{2}{*}{ Treatment factors ${ }^{1}$} & \multicolumn{10}{|c|}{ Cardio-pulmonary morphometry } \\
\hline & $\mathrm{HW}^{2}$ & $\mathrm{LW}^{3}$ & $\mathrm{RVW}^{4}$ & LVW $^{5}$ & $\mathrm{RV} / \mathrm{HW}^{6}$ & $\mathrm{LV} / \mathrm{HW}^{7}$ & $\mathrm{RV} / \mathrm{TV}^{8}$ & $\mathrm{RVT}^{9}$ & RVD $^{10}$ & $\mathrm{HB}^{11}$ \\
\hline \multicolumn{11}{|c|}{$\mathrm{N}_{0} \mathrm{~A}_{1} \mathrm{~V}_{4}=\mathrm{NaHCO}_{3} \mathrm{O} \%$, L-arginine $0.05 \%+$ Vitamin-C $0.05 \%$, Vegetable oil $4 \%$} \\
\hline \multicolumn{11}{|c|}{$\mathrm{N}_{1} \mathrm{~A}_{0} \mathrm{~V}_{4}=\mathrm{NaHCO}_{3} 0.1 \%$, L-arginine $0 \%+$ Vitamin-C $0 \%$, Vegetable oil $4 \%$} \\
\hline \multicolumn{11}{|c|}{$\mathrm{N}_{1} \mathrm{~A}_{1} \mathrm{~V}_{4}=\mathrm{NaHCO}_{3} 0.1 \%$, L-arginine $0.05 \%+$ Vitamin-C $0.05 \%$, Vegetable oil $4 \%$} \\
\hline \multicolumn{11}{|c|}{$\begin{array}{l}{ }^{2} \mathrm{HW}=\text { Heart weight }(\mathrm{g}),{ }^{3} \mathrm{LW}=\mathrm{Lung} \text { weight }(\mathrm{g}),{ }^{4} \mathrm{RVW}=\mathrm{Right} \text { ventricle weight }(\mathrm{g}),{ }^{5} \mathrm{LVW}=\mathrm{Left} \text { ventricle weight }(\mathrm{g}),{ }^{6} \mathrm{RV} / \mathrm{HW}= \\
\text { Right ventricle weight (\% heart weight), }{ }^{7} \mathrm{LV} / \mathrm{HW}=\mathrm{Left} \text { ventricle weight }\left(\% \text { heart weight), }{ }^{8} \mathrm{RV} / \mathrm{TV}=\mathrm{Right} \text { ventricle weight: total }\right. \\
\text { ventricle weight, }{ }^{9} \mathrm{RVT}=\text { Right ventricle thickness }(\mathrm{mm}),{ }^{10} \mathrm{RVD}=\text { Right ventricle diameter }(\mathrm{mm}),{ }^{11} \mathrm{HB}=\text { Heart beat } / \text { minute; }\end{array}$} \\
\hline \multicolumn{11}{|c|}{${ }^{12}$ SEM = Standard error of the means; } \\
\hline \multicolumn{11}{|c|}{${ }^{13} \mathrm{NS}=$ Non-significant $(p>0.05), * \star=$ Significant $(p<0.01)$} \\
\hline
\end{tabular}

\section{Hemato-biochemical indices}

Dietary $\mathrm{NaHCO}_{3}$ increased $(p<0.05)$ TP and vegetable oil increased $(p<0.05) \mathrm{Hb}$ and PCV $(p<0.05)$ as the main effect. The influence of L-arginine + vitamin-C appeared nil $(\mathrm{p}>0.05)$ on all the hemato-biochemical indices. The $\mathrm{NaHCO}_{3} \times \mathrm{L}$-arginine + vitamin-C $\times$ Vegetable oil additively interacted to increase $(p<0.05)$ the triiodothyronine $($ T3) $($ Table 7$)$. 
Table 7

Blood $\mathrm{p}^{\mathrm{H}}$, blood glucose $(\mathrm{mg} / \mathrm{dl})$, total cholesterol (TC, $\left.\mathrm{mg} / \mathrm{dl}\right)$, serum glutamic pyruvic transaminase (SGPT,

$\mathrm{U} / \mathrm{L})$, serum glutamic oxaloacetic transaminase (SGOT, U/L), albumin (mg/dl), total protein (TP, $\mathrm{mg} / \mathrm{dl}$ ),

triiodothyronine $\left(T_{3}, n g / d l\right)$, haemoglobin $(\mathrm{Hb}, \mathrm{g} / \mathrm{dl})$ and packed cell volume (PCV, \%) of the broiler birds fed diets supplemented with various levels of $\mathrm{NaHCO}_{3}$, L-arginine + vitamin- $\mathrm{C}$ and vegetable oils

\begin{tabular}{|c|c|c|c|c|c|c|c|c|c|}
\hline \multirow[t]{2}{*}{ Treatment factors ${ }^{1}$} & \multicolumn{9}{|c|}{ Hemato-biochemical indices } \\
\hline & $\begin{array}{l}\text { Blood } \\
\mathrm{pH}\end{array}$ & $\begin{array}{l}\text { Glucose } \\
\text { (mg/dl) }\end{array}$ & $\begin{array}{l}\text { TC } \\
(\mathrm{mg} / \mathrm{dl})\end{array}$ & $\begin{array}{l}\text { SGPT } \\
(\mathrm{U} / \mathrm{L})\end{array}$ & $\begin{array}{l}\text { SGOT } \\
(\mathrm{U} / \mathrm{L})\end{array}$ & $\begin{array}{l}\text { TP } \\
(g / L)\end{array}$ & $\begin{array}{l}\mathrm{T}_{3} \\
\text { (ng/dl) }\end{array}$ & $\begin{array}{l}\mathrm{Hb} \\
(\mathrm{g} / \mathrm{dl})\end{array}$ & $\begin{array}{l}\text { PCV } \\
(\%)\end{array}$ \\
\hline \multicolumn{10}{|l|}{$\mathrm{NaHCO}_{3}(\mathrm{~N})$} \\
\hline $0.00 \%$ & 6.7 & 259.3 & 141.8 & 14.6 & 23.7 & $32.8^{a}$ & 96.0 & 14.5 & 31.4 \\
\hline $0.10 \%$ & 6.8 & 289.5 & 146.3 & 14.2 & 23.3 & $40.4^{b}$ & 108.0 & 14.5 & 31.2 \\
\hline \multicolumn{10}{|c|}{ L-arginine + vitamin-C $(A)$} \\
\hline $0.00 \%$ & 6.7 & 257.5 & 145.2 & 13.8 & 24.0 & 37.5 & 105.0 & 14.8 & 31.8 \\
\hline $0.10 \%$ & 6.8 & 291.3 & 152.8 & 15.0 & 23.0 & 35.7 & 98.0 & 14.3 & 30.9 \\
\hline \multicolumn{10}{|l|}{ Vegetable oil (V) } \\
\hline $3.00 \%$ & 6.8 & 272.4 & 147.0 & 14.7 & 23.6 & 37.8 & 94.0 & $13.0^{\mathrm{a}}$ & $29.1^{\mathrm{a}}$ \\
\hline $4.00 \%$ & 6.8 & 276.4 & 151.1 & 14.2 & 23.4 & 35.4 & 106.0 & $16.0^{\mathrm{b}}$ & $33.6^{\mathrm{b}}$ \\
\hline \multicolumn{10}{|l|}{$\mathrm{N} \times \mathrm{A} \times \mathrm{V}$} \\
\hline $\mathrm{N}_{0} \times \mathrm{A}_{0} \times \mathrm{V}_{3}$ & 6.7 & 244.9 & 142.1 & 13.2 & 23.0 & 38.7 & 92.0 & 13.3 & 30.3 \\
\hline $\mathrm{N}_{0} \times \mathrm{A}_{1} \times \mathrm{V}_{3}$ & 6.8 & 263.1 & 148.0 & 12.9 & 22.1 & 29.8 & 95.0 & 12.2 & 27.3 \\
\hline $\mathrm{N}_{0} \times \mathrm{A}_{0} \times \mathrm{V}_{4}$ & 6.8 & 237.5 & 140.0 & 15.1 & 26.3 & 28.4 & 91.0 & 17.7 & 35.2 \\
\hline $\mathrm{N}_{0} \times \mathrm{A}_{1} \times \mathrm{V}_{4}$ & 6.6 & 291.6 & 137.0 & 17.4 & 23.2 & 34.4 & 104.0 & 15.0 & 32.9 \\
\hline $\mathrm{N}_{1} \times \mathrm{A}_{0} \times \mathrm{V}_{3}$ & 6.7 & 261.3 & 147.7 & 16.1 & 24.9 & 47.3 & 129.0 & 12.6 & 28.4 \\
\hline$N_{1} \times A_{1} \times V_{3}$ & 6.9 & 320.2 & 150.2 & 16.5 & 24.5 & 35.2 & 74.0 & 13.9 & 30.4 \\
\hline \multicolumn{10}{|c|}{${ }^{1} \mathrm{~N}_{0} \mathrm{~A}_{1} \mathrm{~V}_{3}=\mathrm{NaHCO}_{3} 0 \%$, L-arginine $0.05 \%+$ Vitamin-C $0.05 \%$, Vegetable oil $3 \% ;$} \\
\hline \multicolumn{10}{|c|}{$\mathrm{N}_{1} \mathrm{~A}_{0} \mathrm{~V}_{3}=\mathrm{NaHCO}_{3} 0.1 \%$, L-arginine $0 \%+$ Vitamin-C $0 \%$, vegetable oil $3 \%$; } \\
\hline \multicolumn{10}{|c|}{$\mathrm{N}_{1} \mathrm{~A}_{1} \mathrm{~V}_{3}=\mathrm{NaHCO}_{3} 0.1 \%$, L-arginine $0.05 \%+$ Vitamin-C $0.05 \%$, Vegetable oil $3 \%$; } \\
\hline \multicolumn{10}{|c|}{$\mathrm{N}_{0} \mathrm{~A}_{0} \mathrm{~V}_{4}=\mathrm{NaHCO}_{3} 0 \%$, L-arginine $0 \%+$ Vitamin-C $0 \%$, Vegetable oil $4 \%$; } \\
\hline \multicolumn{10}{|c|}{$\mathrm{N}_{0} \mathrm{~A}_{1} \mathrm{~V}_{4}=\mathrm{NaHCO}_{3} 0 \%$, L-arginine $0.05 \%+$ Vitamin-C $0.05 \%$, Vegetable oil $4 \%$; } \\
\hline \multicolumn{10}{|c|}{$\mathrm{N}_{1} \mathrm{~A}_{0} \mathrm{~V}_{4}=\mathrm{NaHCO}_{3} 0.1 \%$, L-arginine $0 \%+$ Vitamin-C $0 \%$, Vegetable oil $4 \%$; } \\
\hline \multicolumn{10}{|c|}{$\mathrm{N}_{1} \mathrm{~A}_{1} \mathrm{~V}_{4}=\mathrm{NaHCO}_{3} 0.1 \%$, L-arginine $0.05 \%+$ Vitamin-C $0.05 \%$, Vegetable oil $4 \%$; } \\
\hline \multicolumn{10}{|c|}{${ }^{2} \mathrm{SEM}=$ Standard error of the means; } \\
\hline \multicolumn{10}{|c|}{${ }^{3} N S=$ Non-significant $(p>0.05), *=$ Significant $(p<0.05)$} \\
\hline
\end{tabular}




\begin{tabular}{|c|c|c|c|c|c|c|c|c|c|}
\hline \multirow[t]{2}{*}{ Treatment factors $^{1}$} & \multicolumn{9}{|c|}{ Hemato-biochemical indices } \\
\hline & $\begin{array}{l}\text { Blood } \\
\mathrm{pH}\end{array}$ & $\begin{array}{l}\text { Glucose } \\
\text { (mg/dl) }\end{array}$ & $\begin{array}{l}\mathrm{TC} \\
(\mathrm{mg} / \mathrm{dl})\end{array}$ & $\begin{array}{l}\text { SGPT } \\
(U / L)\end{array}$ & $\begin{array}{l}\text { SGOT } \\
(\mathrm{U} / \mathrm{L})\end{array}$ & $\begin{array}{l}\text { TP } \\
(\mathrm{g} / \mathrm{L})\end{array}$ & $\begin{array}{l}\mathrm{T}_{3} \\
\text { (ng/dl) }\end{array}$ & $\begin{array}{l}\mathrm{Hb} \\
(\mathrm{g} / \mathrm{dl})\end{array}$ & $\begin{array}{l}\text { PCV } \\
\text { (\%) }\end{array}$ \\
\hline $\mathrm{N}_{1} \times \mathrm{A}_{0} \times \mathrm{V}_{4}$ & 6.7 & 286.2 & 151.2 & 10.9 & 21.9 & 35.5 & 107.0 & 15.3 & 33.2 \\
\hline $\mathrm{N}_{1} \times \mathrm{A}_{1} \times \mathrm{V}_{4}$ & 7.0 & 290.3 & 176.1 & 13.4 & 22.0 & 43.4 & 121.0 & 16.1 & 32.9 \\
\hline SEM $^{2}$ & 0.07 & 9.54 & 4.05 & 0.72 & 5.82 & 1.82 & 4.6 & 0.69 & 1.01 \\
\hline \multicolumn{10}{|l|}{ Significance ${ }^{3}$} \\
\hline $\mathrm{NaHCO}_{3}$ & NS & NS & NS & NS & NS & * & NS & NS & NS \\
\hline Additives & NS & NS & NS & NS & NS & NS & NS & NS & NS \\
\hline Vegetable oil & NS & NS & NS & NS & NS & NS & NS & * & * \\
\hline NaHCO3×L-arg.xVeg. & NS & NS & NS & NS & NS & NS & * & NS & NS \\
\hline \multicolumn{10}{|c|}{${ }^{1} \mathrm{~N}_{0} \mathrm{~A}_{1} \mathrm{~V}_{3}=\mathrm{NaHCO}_{3} 0 \%$, L-arginine $0.05 \%+$ Vitamin-C $0.05 \%$, Vegetable oil $3 \%$; } \\
\hline \multicolumn{10}{|c|}{$\mathrm{N}_{1} \mathrm{~A}_{0} \mathrm{~V}_{3}=\mathrm{NaHCO}_{3} 0.1 \%$, L-arginine $0 \%+$ Vitamin-C $0 \%$, vegetable oil $3 \%$; } \\
\hline \multicolumn{10}{|c|}{$\mathrm{N}_{1} \mathrm{~A}_{1} \mathrm{~V}_{3}=\mathrm{NaHCO}_{3} 0.1 \%$, L-arginine $0.05 \%+$ Vitamin-C $0.05 \%$, Vegetable oil $3 \% ;$} \\
\hline \multicolumn{10}{|c|}{$\mathrm{N}_{0} \mathrm{~A}_{0} \mathrm{~V}_{4}=\mathrm{NaHCO}_{3} 0 \%$, L-arginine $0 \%+$ Vitamin-C $0 \%$, Vegetable oil $4 \%$} \\
\hline \multicolumn{10}{|c|}{$\mathrm{N}_{0} \mathrm{~A}_{1} \mathrm{~V}_{4}=\mathrm{NaHCO}_{3} 0 \%$, L-arginine $0.05 \%+$ Vitamin-C $0.05 \%$, Vegetable oil $4 \%$} \\
\hline \multicolumn{10}{|c|}{$\mathrm{N}_{1} \mathrm{~A}_{0} \mathrm{~V}_{4}=\mathrm{NaHCO}_{3} 0.1 \%$, L-arginine $0 \%+$ Vitamin-C $0 \%$, Vegetable oil $4 \%$; } \\
\hline \multicolumn{10}{|c|}{$\mathrm{N}_{1} \mathrm{~A}_{1} \mathrm{~V}_{4}=\mathrm{NaHCO}_{3} 0.1 \%$, L-arginine $0.05 \%+$ Vitamin-C $0.05 \%$, Vegetable oil $4 \% ;$} \\
\hline \multicolumn{10}{|c|}{${ }^{2}$ SEM = Standard error of the means; } \\
\hline \multicolumn{10}{|c|}{${ }^{3} N S=$ Non-significant $(p>0.05), *=$ Significant $(p<0.05)$} \\
\hline
\end{tabular}

\section{Discussion}

According to Wideman et al. (2013), the left ventricle of the heart drives the oxygenated blood to the whole body to support maintenance, growth and productivity of the broiler birds. The volume of blood pumped by the left ventricle each minute is known as the cardiac output which in clinically healthy bird is propelled entirely by the right ventricle through the lungs at relatively low pulmonary arterial pressure to reduce the risk of fluid filtration into the gas exchange spaces (Martinez-Lemus et al., 1999, 2003; Odom et al., 2004; Wideman et al., 2013). This low pressure sustains as long as pulmonary vasculature upholds a suitably low resistance to the blood flow. However, genetically modified broiler birds with improved growth potentials, feed efficiency and relatively higher muscle to organ ratio require increased blood flow to satisfy their ever increasing metabolic demand. In order to supply increased blood flow, the right ventricle consistently initiate elevated heart beats leading to tachycardia.

Under critical episodes of progressive tachycardia, the pulmonary arterial pressure accelerates the rate of blood flow which stimulates RBC racing too fast through the pulmonary vasculature (Wideman et al., 2013). Due to increased circulation rate, RBC gets shorter than the normal transit time at the gas exchange surfaces for complete diffusive exchange of $\mathrm{O}_{2}$ and $\mathrm{CO}_{2}$ and fails to achieve full blood-gas equilibrium (Henry and Fedde, 1970; Powell et al., 2000; Wideman et al., 2013). The unsaturated blood 
then enters the systemic flow with a lower than usual partial pressure of $\mathrm{O}_{2}$ and a higher partial pressure of $\mathrm{CO}_{2}$ resulting hypoxemia (Peacock et al. 1990; Reeves et al. 1991; Julian and Mirsalimi 1992; Wideman and Kirby 1996; Roush et al. 1997).

Hypoxemia further triggers polycythemia by stimulating erythropoietin which increases hematocrit to enhance the $\mathrm{O}_{2}$ carrying capacity of blood (Shlosberg et al., 1996; Scheele et al., 2003; Tekeli, 2014). Progressive elevation of hematocrit and the reduced deformability of the rapidly produced immature erythrocytes consistently increase blood viscosity (Gregersen et al., 1969; Hakim and Macek, 1988), thereby, thrombotic occlusion (Currie, 1999; Baghbanzadeh and Decuypere, 2008; Wideman et al., 2013) and finally ascitic heart failure (Fedde and Wideman 1996; Shlosberg et al. 1996, 1998; Wideman et al. 1998). Consistent tachycardia as well as polycythemia thus indirectly bears indications of ascites which impairs performance, carcass and health of the birds as measured by hemato-biochemical indices.

\section{Performance}

Our study indicated that, the addition of L-arginine + vitamin-C in the diet of the broiler chicken significantly improved feed intake from 3-5 weeks. Similar result was reported by Al-Daraji and Salih (2012). Supplementation of L-arginine + vitamin-C in the mash feed resulted better weight gain during last two weeks of rearing which is in line with the findings of other studies (Njoku 1986; Al-Daraji and Salih 2012). Significantly higher weight gain might have been due to increased feed intake. It is well established that, vitamin-C reduces the extents of oxidative heat stress efficiently (Masad 2012; Dalia et al. 2018) which is the underlying cause of increased feed intake and weight gain (Njoku, 1986) in the broiler birds in our study. Further, these two additives improve the immune status of the birds which could have helped in better performance of the commercial broiler birds used in current study (Masad 2012; Dalia et al. 2018). Similarly, L-arginine, an indispensable amino acid for poultry as well as human which improves the release of growth hormone and muscle growth that could have contributed to the better feed intake and daily weight gain in our study (Stevens et al. 2000).

In the present study, incorporation of vegetable oil in the diet of boiler birds significantly improved average daily gain and FCR during the initial period. These findings coincide the results of Attia et al. (2020) although differ from the observation of Ayed et al. (2015). One of the objectives of the current study was to see the interaction effect of $\mathrm{NaHCO}_{3}, \mathrm{~L}$-arginine + vitamin-C and vegetable oil in accordance with the performance of broiler chicken. Interaction effect of $\mathrm{NaHCO}_{3}$ and L-arginine + vitamin-C showed significant rise in feed intake in the last two weeks of the trial. As stated above, effect of reducing heat stress due to addition of additives present in the diet could be one of the reasons behind the improved feed intake (Masad 2012; Dalia et al. 2018). Accordingly, at the same time, $\mathrm{NaHCO}_{3}$ on diet apparently had association with the increasing feed intake and the reason appeared to be due to the bicarbonate ion which was associated with an increased water intake (Balnave and Gorman, 1993). Similar interaction effects were seen in the birds fed diet containing combination of L-arginine + vitamin-C and vegetable oil. As mentioned earlier, vitamin-C in the test diets helped to reduce the environmental stress (Masad 2012; Dalia et al. 2018). Further, the vegetable oils in the diet met the increasing demand of energy (Attia et al., 2020), especially during the finisher stage. These effects might be the reason of rise in energy intake in the broiler birds fed diets containing both the L-arginine + vitamin-C and the vegetable oil.

From the result of our study, it is obvious that, average feed intake of the birds fed $\mathrm{NaHCO}_{3}$ supplemented diet throughout the trial increased substantially which agrees with the previous findings (Arp et al., 1984; Roussan et al., 2008; Osman et al., 2015). The increased feed intake, however, might be a result of reduced heat stress in broilers caused by $\mathrm{NaHCO}_{3}$ (Roussan et al., 2008). In our study, $\mathrm{NaHCO}_{3}$ also improved the average daily gain of birds in the last two weeks which is aligned with the previous studies (Roussan et al., 2008; Osman et al., 2015; Saker et al., 2020). Better feed intake and daily gain of broilers fed $\mathrm{NaHCO}_{3}$ ultimately resulted significantly better FCR in the last two weeks which is supported by the previous studies (Hooge et al., 2000; Nidgundi et al., 2007).

\section{Carcass characteristics}

All the carcass criteria, i.e., dressing percentage, weight of breast, thigh, wings, drumstick and other organs were unaffected among dietary treatments in the current study. These findings agree with the results of some previous studies (Petrolli et al., 2016) although differ with the results reported by others elsewhere (Hooge et al., 1999; Ogunwole et al., 2014). Here, in our study,

Page 20/27 
liver weight was found lower in broiler birds fed experimental diets containing L-arginine + vitamin-C that further coincides with the findings of Susantoputro et al. (2014). The interaction effects of test ingredients on carcass merit were also found nonsignificant between diets.

\section{Cardio-pulmonary morphometry}

Dietary supplementation of $130 \%$ arginine of the requirement improved the intestinal morphology and performance and decreased the cold induced ascitic mortality in broiler chickens (Abdulkarimi et al., 2019). In a previous study, additional arginine administered in ovo or in the feed reduced the vulnerability of broilers to pulmonary hypertension (Saki et al., 2013). Additionally, broiler chickens reared at high altitude and predisposed to pulmonary hypertension and ascites were partly explained by arginine supplementation (Khajali et al., 2011). Supplemental arginine improved the pulmonary vascular performance of hypoxic broiler birds and its outcomes were further enhanced by the addition of the vitamin-C. Arginine and antioxidant vitamins might have taken part in synergistic functions to improve nitric oxide bioavailability as potent natural vasodilator and lessen oxidative damage, thus increasing cardiopulmonary performance (Bautista-Ortega and Ruiz-Feria, 2010).

It was further reported that, arginine or guanidinoacetic acid supplementation of diets did not affect gross response of birds under standard temperature, but addition of arginine to the diet significantly reduced the incidence of cold stress on performance, gut development and ascites syndrome (Kodambashi et al. 2017). In fact, L-arginine is a substrate for nitric oxide, which acts as a potent endogenous pulmonary vasodilator that substantially reduces the right ventricle: total ventricle ratio (Wideman et al. 1995). Consistent with these points, addition of L-arginine and thereby, reduced susceptibility of the birds against tachycardia and polycythemia evident in our study is likely.

Rostami et al. (2016) reported that, the birds receiving flax oil had significantly higher serum concentration of nitric oxide. The right-to-total ventricle weight ratio (RV/TV) and death from pulmonary hypertension were significantly $(p<0.05)$ declined in birds that fed on flax oil. It was argued that, n-3 fatty acids could significantly lessen RV:TV and PHS death in birds by escalating circulatory level of nitric oxide and suppressing hepatic lipogenesis. In another study, administration of flax oil reduced blood viscosity, right ventricular hypertrophy, hematocrit and hemoglobin content and improved erythrocyte deformability by increasing the quantity of unsaturated fatty acids in the erythrocyte membranes and thereby decreased ascites induced mortality(Walton et al., 1999, 2001). With reference to these points, addition of vegetable oil in the present study might have contributed additional n3 fatty acids responsible for improving RV:TV and thereby no evidence of tachycardia in the experimental birds fed test diets in our study.

Supplementation of vitamin C substantially improved cellular integrity and reduced incidence of mortality by ascites in a previous study (Roch et al., 2000). Similarly, addition of vitamin C reduced the possibility of thick-walled peripheral vessels in the lungs and thereby, the incidence of ascites (Xiang et al., 2002). Vitamin-C reduces muscularization of the pulmonary arterioles by scavenging oxygen-derived free radicals thus lower the number of thick-walled peripheral vessels to decrease the resistance to blood flow in the pulmonary vessels of broilers (Hassanzadeh Ladmakhi et al., 1997). The levels of a-tocopherol and $y$-tocopherol were decreased in the mitochondria of an ascitic broiler, suggesting inadequate reactive oxygen scavenger in the primary site of energy transduction (Cawthorn et al., 2001). It could therefore be inferred that, addition of L-arginine + vitamin-C in the form of feed additive efficiently stabilized RV:TV, prevented tachycardia and polycythemia in the experimental birds used in our study.

\section{Hemato-biochemical indices}

A decline in blood $\mathrm{pH}$ lowers the oxygen affinity of haemoglobin, while increased blood pH increases oxygen affinity to increase haemoglobin saturation in the lungs (Isaacks et al., 1986). Thus, increased blood pH can improve the loading capacity of oxygen by haemoglobin in the lungs due to the Bohr effect. Accordingly, feeding bicarbonate supplemented diets result in a decrease in pulmonary hypertension (Barer et al. 1966; Balnave and Gorman 1993; Squires and Julian 2001). Perhaps, this is the reason behind the absence of tachycardia and thereby polycythemia in the experimental birds used in the present study.

The majority of commercial meat birds are fed crumbled or pelleted diets to attain utmost growth and feed efficiency. Feeding mash diet reduces growth rate. Broiler birds that consumed pellet feed had frequently been shown to have higher incidence of tachycardia than the birds that consumed the same diet in mash form (Shlosberg et al., 1992; Bölükbasi et al., 2005). Unlike

Page 21/27 
pellets, mash diets are not subjected to steam conditioning prior to pelleting. Thus, mash diets are less palatable and have reduced microbial and enzymatic digestibility. Consequently, mash diets have fur less susceptibility for tachycardia and polycythemia in broiler birds as evident in our study.

In ascitic birds, Dahiya et al. (2001) reported a significant rise in haemoglobin, packed cell volume, total erythrocyte count, serum alanine aminotransferase, asparate aminotransferase, alkaline phosphates and phosphorus and marked fall in serum total proteins, albumin globulin ratio (A:G), calcium and sodium although erythrocyte indices, i.e., mean corpuscular volume, and mean corpuscular haemoglobin concentration remained unchanged. Consistent results were reported elsewhere indicating significant increase in haemoglobin concentration (Maxwell et al., 1986; Dahiya et al., 2001; Ipek and Sahan, 2006; Wang et al., 2012; Tekeli, 2014), higher values for haematocrit and total erythrocyte count (Maxwell et al. 1986; Dahiya et al. 2001; Ipek and Sahan 2006; Reza et al. 2008; Hafshejani et al. 2012; Wang et al. 2012; Tekeli 2014) in ascitic birds.

Similarly, marked decrease in plasma proteins, i.e., total proteins, albumin and albumin:globulin ratio (Biswas et al., 1995; Dahiya et al., 2001; Daneshyar et al., 2009; Wang et al., 2012) and increase in serum bilirubin, AST, ALT, LDH, ALP (Dahiya et al., 2001;

Reza et al., 2008), plasma lipids and total cholesterol (Biswas et al., 1995; Wang et al., 2012) were reported further in ascitic birds. Additionally, the HDL cholesterol significantly decreased reflecting progressive cardiomyopathy and subsequently cardiac failure in previous studies. Since direct evidences are scant, relying upon above reports, it can indirectly be inferred that the birds susceptible for tachycardia and polycythemia will reveal similar symptoms. Interestingly, all above hemato-biochemical parameters as well as heart beat persisted within standard range (Bounous and Stedman, 2000) exhibiting no unusual changes thereby least possibility of tachycardia or polycythemia either in control or test groups in our study.

\section{Conclusion}

Plant based high energy mash diets are not susceptible to tachycardia and polycythemia. Addition of $\mathrm{NaHCO}_{3}$, L-arginine+ Vitamin-C ameliorate propensity of tachycardia and polycythemia without deteriorating performance, carcass characteristics and hemato-biochemical indices of the commercial broiler birds in a dose dependent manner.

\section{Declarations}

\section{Funding}

The authors acknowledge 'The University Grants Commission, Bangladesh' for providing research grant.

\section{Conflicts of interest/Competing interests}

None.

\section{Availability of data and materials}

All the data used in the manuscript exclusively belongs to the mentioned authors.

\section{Code availability}

None.

\section{Author's contribution}

Dr. Md. Emran Hossain conceived the study, analyzed data, interpreted the results and finalized the draft. Dr. Nasima Akter procured research grant, conducted the animal trial, ran the immunization program, collected data and prepared the initial draft. All authors read and approved the final manuscript.

\section{Ethics approval}


The entire experimental protocol was approved by the animal welfare law in Bangladesh (Memo No.

CVASU/Dir(R\&E)EC/2021/244(3).

\section{Consent for publication}

Yes.

\section{Consent to participate}

Yes.

\section{Acknowledgement}

The "MAS Additives" (http:// additivesmas.com/ index. html) provided the emulsifiers.

\section{References}

1. Abdulkarimi, R., Shahir, M.H. and Daneshyar, M., 2019. Effects of dietary glutamine and arginine supplementation on performance, intestinal morphology and ascites mortality in broiler chickens reared under cold environment, AsianAustralasian journal of animal sciences, 32, 110.

2. Al-Daraji, H.J. and Salih, A.M., 2012. Effect of dietary L-arginine on productive performance of broiler chickens, Pakistan Journal of Nutrition, 11, 252.

3. Al-Masad, M., 2012. Effects of vitamin C and zinc on broilers performance of immunocompetence under heat stress., Asian Journal of Animal Sciences, 6, 76-84.

4. AOAC, 2019. Official methods of analysis of the AOAC (Association of the Official Agricultural Chemists) International, 21 st edn., Rockville, Maryland, USA.

5. Arp, S.C., Owen, F.N. and Teeter, R.G., 1984. The influence of dietary niacin, ascorbic acid, and sodium bicarbonate on alleviating heat stress in broiler chicks, Oklahoma Agricultural Experiment Station Report, pp. 56-58.

6. Attia, Y.A., Al-Harthi, M.A. and El-Maaty, H.M.A., 2020. The Effects of Different Oil Sources on Performance, Digestive Enzymes, Carcass Traits, Biochemical, Immunological, Antioxidant, and Morphometric Responses of Broiler Chicks, Frontiers in Veterinary Science, 7, 1-12.

7. Ayed, H. Ben, Attia, H. and Ennouri, M., 2015. Effect of oil supplemented diet on growth performance and meat quality of broiler chickens, Advanced Techniques in Biology \& Medicine, 4, 156.

8. Baghbanzadeh, A. and Decuypere, E., 2008. Ascites syndrome in broilers: physiological and nutritional perspectives, Avian pathology, 37, 117-126.

9. Balnave, D. and Gorman, I., 1993. A role for sodium bicarbonate supplements for growing broilers at high temperatures, World's Poultry Science Journal, 49, 236-241.

10. Barer, G.R., Howard, P. and Marlow, J.R., 1966. Effect of hypercapnia and blood pH on pulmonary circulation, Journal of Physiology-London, 182, 29.

11. Bautista-Ortega, J. and Ruiz-Feria, C.A., 2010. L-Arginine and antioxidant vitamins $E$ and C improve the cardiovascular performance of broiler chickens grown under chronic hypobaric hypoxia, Poultry Science, 89, 2141-2146.

12. Biswas, N.K., Dalapati, M.R. and Bhowmik, M.K., 1995. Ascites syndrome in broiler chicken: Observations on certain biochemical and pathological changes, The Indian Journal of Animal Sciences, 65, 1068-1072.

13. Bölükbasi, S.C., Aktas, M.S. and Güzel, M., 2005. The Effect of Feed Regimen on Ascites Induced by Cold Temperatures, International Journal of Poultry Science, 4, 326-329.

14. Bounous, D. and Stedman, N., 2000. Normal avian hematology: chicken and turkey. In: Feldman BF, Zinkl JG, Jain NC, editors. Schalm's veterinary hematology, Wiley, New York, pp.1147-1154.

15. Burton, R.R., Sahara, R. and Smith, A.H., 1971. The hematology of domestic fowl native to high altitude, Environmental Physiology, 1, 155-163. 
16. Cawthorn, D., Beers, K. and Bottje, W.G., 2001. Electron transport chain defect and inefficient respiration may underlie pulmonary hypertension syndrome (ascites)-associated mitochondrial dysfunction in broilers, Poultry Science, 80, 474-484.

17. Currie, R.J.W., 1999. Ascites in poultry: recent investigations, Avian pathology, 28, 313-326.

18. Dahiya, J.P., Bratia, K.C., Mahajan, N.K. and Mishra, S.K., 2001. Blood indices and serum biochemical profile in ascites syndrome affected broilers, Indian Journal of Poultry Science, 36, 33-36.

19. Dalia, A.M., Loh, T.C., Sazili, A.Q., Jahromi, M.F. and Samsudin, A.A., 2018. Effects of vitamin E, inorganic selenium, bacterial organic selenium, and their combinations on immunity response in broiler chickens, BMC veterinary research, 14, 249.

20. Daneshyar, M., Kermanshahi, H. and Golian, A., 2009. Changes of biochemical parameters and enzyme activities in broiler chickens with cold-induced ascites, Poultry Science, 88, 106-110.

21. Decuypere, E., Buyse, J. and Buys, N., 2000. Ascites in broiler chickens: exogenous and endogenous structural and functional causal factors, World's poultry science journal, 56, 367-377.

22. Duncan, D.B., 1955. Multiple Range and Multiple F Tests, Biometrics, 11, 1.

23. Fedde, M.R. and Wideman Jr, R.F., 1996. Blood viscosity in broilers: influence on pulmonary hypertension syndrome, Poultry Science, 75, 1261-1267.

24. Gregersen, M.I., Bryant, C.A., Chien, S., Dellenback, R.J., Magazinovic, V. and Usami, S., 1969. Species differences in the flexibility and deformation of erythrocytes (RBC)., Bibliotheca anatomica, 10, 104.

25. Hafshejani, E.F., Gholami-Ahangaran, M. and Hosseni, E., 2012. Study of blood cells, blood gases and thyroid hormones in broiler chickens suspected of ascites Syndrome, Global Veterinaria, 8, 18-21.

26. Hakim, T.S. and Macek, A.S., 1988. Effect of hypoxia on erythrocyte deformability in different species, Biorheology, 25, 857868.

27. Hassanzadeh Ladmakhi, M., Buys, N., Dewil, E., Rahimi, G., Decuypere, E., Ladmakhi, M.H., Buys, N., Dewil, E., Rahimi, G. and Decuypere, E., 1997. The prophylactic effect of vitamin C supplementation on broiler ascites incidence and plasma thyroid hormone concentration, Avian Pathology, 26, 33-44.

28. Henry, J.D. and Fedde, M.R., 1970. Pulmonary circulation time in the chicken, Poultry science, 49, 1286-1290.

29. Hooge, D., Cummings, K. and McNaughton, J., 2000. Effects of an lonophore Coccidiostat (Monensin or Salinomycin), Sodium or Potassium Bicarbonate, or Both, and Bacitracin Methylene Disalicylate in Broiler Chicken Diets, Revista Brasileira de Ciência Avícola, 2, 75-83.

30. Hooge, D.M., Cummings, K.R., McNaughton, J.L., Quarles, C.L. and George, B.A., 1999. Dietary Sodium Bicarbonate, Coccidial Challenge, and lonophore Coccidiostats in Broiler Chickens, Journal of Applied Poultry Research, 8, 89-99.

31. Ipek, A. and Sahan, U., 2006. Effects of cold stress on broiler performance and ascites susceptibility, Asian-Australasian Journal of Animal Sciences, 19, 734-738.

32. Isaacks, R., Goldman, P. and Kim, C., 1986. Studies on avian erythrocyte metabolism. XIV. Effect of CO2 and pH on P50 in the chicken, American Journal of Physiology-Regulatory, Integrative and Comparative Physiology, 250, R260-R266.

33. Jones, R., 1984. A standard method of dissection of poultry for carcass analysis, Technical Bulletin No. 222, West of Scotland Agricultural College, Scotland., Technical Bulletin. 222.

34. Julian, R.J. and Mirsalimi, S.M., 1992. Blood oxygen concentration of fast-growing and slow-growing broiler chickens, and chickens with ascites from right ventricular failure, Avian diseases, 36, 730-732.

35. Julien, M., Hoeffel, J.M. and Flick, M.R., 1985. Oleic acid lung injury in sheep, Journal of Applied Physiology, 60, 433-440.

36. Khajali, F., Tahmasebi, M., Hassanpour, H., Akbari, M.R., Qujeq, D. and Wideman, R.F., 2011. Effects of supplementation of canola meal-based diets with arginine on performance, plasma nitric oxide, and carcass characteristics of broiler chickens grown at high altitude, Poultry Science, 90, 2287-2294.

37. Khajali, F. and Wideman, R.F., 2016. Nutritional approaches to ameliorate pulmonary hypertension in broiler chickens, Journal of animal physiology and animal nutrition, 100, 3-14.

38. Kodambashi Emami, N., Golian, A., Rhoads, D.D. and Danesh Mesgaran, M., 2017. Interactive effects of temperature and dietary supplementation of arginine or guanidinoacetic acid on nutritional and physiological responses in male broiler 
chickens, British poultry science, 58, 87-94.

39. Martinez-Lemus, L.A., Hester, R.K., Becker, E.J., Jeffrey, J.S. and Odom, T.W., 1999. Pulmonary artery endothelium-dependent vasodilation is impaired in a chicken model of pulmonary hypertension, American Journal of Physiology-Regulatory, Integrative and Comparative Physiology, 277, R190-R197.

40. Martinez-Lemus, L.A., Hester, R.K., Becker, E.J., Ramirez, G.A. and Odom, T.W., 2003. Pulmonary artery vasoactivity in broiler and leghorn chickens: An age profile, Poultry Science, 82, 1957-1964.

41. Maxwell, M.H., Robertson, G.W. and Spence, S., 1986. Studies on an ascitic syndrome in young broilers 1 . Haematology and pathology, Avian Pathology, 15, 511-524.

42. Nidgundi, S.A., Mulla, J.A. and Shivakumar, M.C., 2007. Supplementation of sodium bicarbonate on performance of broiler during summer, Indian Journal of Poultry Science, 42, 98-100.

43. Njoku, P.C., 1986. Effect of dietary ascorbic acid (vitamin C) supplementation on the performance of broiler chickens in a tropical environment, Animal Feed Science and Technology, 16, 17-24.

44. Odom, T.W., Martinez-Lemus, L.A., Hester, R.K., Becker, E.J., Jeffrey, J.S., Meininger, G.A. and Ramirez, G.A., 2004. In vitro hypoxia differentially affects constriction and relaxation responses of isolated pulmonary arteries from broiler and leghorn chickens, Poultry Science, 83, 835-841.

45. Ogunwole, O.A., Obo, E.J. and Majekodunmi, B.C., 2014. Chemical composition of bone and meat of broiler chickens given oral aqueous vitamin $C$ and sodium bicarbonate under natural heat stress, International Journal of AgriScience, 4, 420-425.

46. Osman, A.A.B., Hamed, A.H.M., Muna, M.M. and Mysara, S.M., 2015. Effects of Sodium Bicarbonate Levels on the Performance of Broiler Chickens under Sudan Condition, Asian Journal of Agriculture and Food Sciences, 03, 2321-1571.

47. Peacock, A.J., Pickett, C., Morris, K. and Reeves, J.T., 1990. Spontaneous hypoxaemia and right ventricular hypertrophy in fast growing broiler chickens reared at sea level., Comparative biochemistry and physiology. A, Comparative physiology, 97 , 537-541.

48. Penney, D.G., Davidson, S.B., Gargulinski, R.B. and Caldwell-Ayre, T.M., 1988. Heart and lung hypertrophy, changes in blood volume, hematocrit and plasma renin activity in rats chronically exposed to increasing carbon monoxide concentrations, Journal of applied toxicology, 8, 171-178.

49. Petrolli, T.G., Junqueira, O.M., Domingues, C.H.F., Pereira, A.S.C., Santos, E.T. and Rocha, R.X., 2016. Effects of the addition of glucose, sodium bicarbonate, and vitamin $E$ to the drinking water of pre-slaughter broiler chickens on carcass yield, gastric emptying and meat quality, Revista Brasileira de Ciencia Avicola, 18, 29-34.

50. Powell, F.L., Dwinell, M.R. and Aaron, E.A., 2000. Measuring ventilatory acclimatization to hypoxia: Comparative aspects, Respiration Physiology, 122, 271-284.

51. Powell, F.L., Hastings, R.H. and Mazzone, R.W., 1985. Pulmonary vascular resistance during unilateral pulmonary arterial occlusion in ducks, American Journal of Physiology - Regulatory Integrative and Comparative Physiology, 18, 39-43.

52. Reeves, J.T., Ballam, G., Hofmeister, S., Pickett, C., Morris, K. and Peacock, A., 1991. Improved arterial oxygenation with feed restriction in rapidly growing broiler chickens., Comparative biochemistry and physiology. A, Comparative physiology, 99 , 481-485.

53. Reza, J., Arab, H.A., Rasouli, A., Javaheri, A.A. and Reza, S.G., 2008. Biochemical, haematological and pathological alterations associated with ascites in broilers and the role of oxygen-derived free radicals, Journal of Veterinary Research, $\mathbf{6 2}$, 333-339.

54. Roch, G., Boulianne, M. and Roth, L. de, 2000. Dietary antioxidants reduce ascites in broilers., World poultry, 16, 18-22.

55. Rostami, A., Zamani Moghaddam, A.K., Hassanpour, H. and Khajali, F., 2016. Pulmonary hypertension and right ventricular failure in broiler chickens reared at high altitude is affected by dietary source of n-6 and n-3 fatty acids, Journal of Animal Physiology and Animal Nutrition, 100, 701-706.

56. Roush, W.B., Cravener, T.L., Kirby, Y.K. and Wideman Jr, R.F., 1997. Probabilistic neural network prediction of ascites in broilers based on minimally invasive physiological factors, Poultry Science, 76, 1513-1516.

57. Roussan, D.A., Khwaldeh, G.Y., Haddad, R.R., Shaheen, I.A., Salameh, G. and Al Rifai, R., 2008. Effect of ascorbic acid, acetylsalicylic acid, sodium bicarbonate, and potassium chloride supplementation in water on the performance of broiler 
chickens exposed to heat stress, Journal of Applied Poultry Research, 17, 141-144.

58. Ruiz-Feria, C.A. and Wideman Jr, R.F., 2001. Taurine, cardiopulmonary hemodynamics, and pulmonary hypertension syndrome in broilers, Poultry Science, $80,1607-1618$.

59. Saker, O., Dakroury, M., Sokary, E., Risk, W. and Abass, M., 2020. Ameliorative Effect of Dietary Acetylsalicylic Acid and Sodium Bicarbonate Supplementation on Growth and Health Status of broiler chicks exposed to heat-stress, Alexandria Journal of Veterinary Sciences, 64, 54.

60. Saki, A., Haghighat, M. and Khajali, F., 2013. Supplemental arginine administered in ovo or in the feed reduces the susceptibility of broilers to pulmonary hypertension syndrome, British Poultry Science, 54, 575-580.

61. Scheele, C.W., Van Der Klis, J.D., Kwakernaak, C., Buys, N. and Decuypere, E., 2003. Haematological characteristics predicting susceptibility for ascites. 2. High haematocrit values in juvenile chickens, British poultry science, 44, 484-489.

62. Shlosberg, A., Bellaiche, M., Berman, E., Perk, S., Deeb, N., Neumark, E. and Cahaner, A., 1998. Relationship between broiler chicken haematocrit-selected parents and their progeny, with regard to haematocrit, mortality from ascites and bodyweight, Research in veterinary science, 64, 105-109.

63. Shlosberg, A., Bellaiche, M., Zeitlin, G., Ya'Acobi, M. and Cahaner, A., 1996. Hematocrit values and mortality from ascites in cold-stressed broilers from parents selected by hematocrit, Poultry Science, 75, 1-5.

64. Shlosberg, A., Zadikov, I., Bendheim, U., Handji, V. and Berman, E., 1992. The Effects of Poor Ventilation, Low Temperatures, Type of Feed and Sex of Bird On the Development of Ascites in Broilers. Physiopathological Factors, Avian Pathology, 21, 369-382.

65. Snyder, G.K., 1971. Influence of temperature and hematocrit on blood viscosity., The American journal of physiology, 220, 1667-1672.

66. Squires, E.J. and Julian, R.J., 2001. The effect of dietary chloride and bicarbonate on blood pH, haematological variables, pulmonary hypertension and ascites in broiler chickens, British Poultry Science, 42, 207-212.

67. Stevens, B.R., Godfrey, M.D., Kaminski, T.W. and Braith, R.W., 2000. High-intensity dynamic human muscle performance enhanced by a metabolic intervention, Medicine and Science in Sports and Exercise, 32, 2102-2108.

68. Susantoputro, S.H., Arimbi, H.P. and Legowo, D., 2014. Potensi Suplementasi Potasium Klorida dan Sodium Bikarbonat Sebagai Thermotolerance Agent pada Hepar Broiler yang Terpapar Heat Stress Kronis The Potency of Potassium Chloride and Sodium Bicarbonate Suplementation as Thermotolerance Agent on Liver of Chro, Media Kedokteran Hewan, 30, 67-74.

69. Tekeli, A., 2014. Effects of ascites (pulmonary hypertension syndrome) on blood gas, blood oximetry parameters and heart sections of broilers grown at high altitude, Journal of Animal and Plant Sciences, 24, 998-1002.

70. Walton, J.P., Bond, J.M., Julian, R.J. and Squires, E.J., 1999. Effect of dietary flax oil and hypobaric hypoxia on pulmonary hypertension and haematological variables in broiler chickens, British poultry science, 40, 385-391.

71. Walton, J.P., Julian, R.J. and Squires, E.J., 2001. The effects of dietary flax oil and antioxidants on ascites and pulmonary hypertension in broilers using a low temperature model, British Poultry Science, 42, 123-129.

72. Wang, Y., Guo, Y., Ning, D., Peng, Y., Cai, H., Tan, J., Yang, Y. and Liu, D., 2012. Changes of hepatic biochemical parameters and proteomics in broilers with cold-induced ascites, Journal of animal science and biotechnology, 3, 41.

73. Wideman J, R.F., Kirby, Y.K., Tackett, C.D., Marson, N.E., Tressler, C.J. and Mcnew, R.W., 1996. Independent and simultaneous unilateral occlusion of the pulmonary artery and extra-pulmonary primary bronchus in broilers, Poultry science, 75, 14171427.

74. Wideman Jr, R.F. and Kirby, Y.K., 1996. Electrocardiographic evaluation of broilers during the onset of pulmonary hypertension initiated by unilateral pulmonary artery occlusion, Poultry Science, 75, 407-416.

75. Wideman Jr, R.F., Kirby, Y.K., Ismail, M., Bottje, W.G., Moore, R.W. and Vardeman, R.C., 1995. Supplemental L-arginine attenuates pulmonary hypertension syndrome (ascites) in broilers, Poultry Science, 74, 323-330.

76. Wideman Jr, R.F., Kirby, Y.K., Owen, R.L. and French, H., 1997. Chronic unilateral occlusion of an extrapulmonary primary bronchus induces pulmonary hypertension syndrome (ascites) in male and female broilers, Poultry Science, 76, 400-404.

77. Wideman Jr, R.F. and Tackett, C.D., 2000. Cardio-pulmonary function in broilers reared at warm or cool temperatures: effect of acute inhalation of $100 \%$ oxygen, Poultry Science, $79,257-264$.

Page 26/ 27 
78. Wideman Jr, R.F., Wing, T., Kirby, Y.K., Forman, M.F., Marson, N., Tackett, C.D. and Ruiz-Feria, C.A., 1998. Evaluation of minimally invasive indices for predicting ascites susceptibility in three successive hatches of broilers exposed to cool temperatures, Poultry science, $77,1565-1573$.

79. Wideman, R.F., 1999. Cardiac output in four-, five-, and six-week-old broilers, and hemodynamic responses to intravenous injections of epinephrine, Poultry Science, 78, 392-403.

80. Wideman, R.F., 2000. Cardio-pulmonary hemodynamics and ascites in broiler chickens, Poultry and Avian Biology Reviews, $11,21-44$.

81. Wideman, R.F. and Bottje, W.G., 1993. Current understanding of the ascites syndrome and future research directions, In: Nutrition and Technical Symposium Proceedings. Novus International, Inc., St. Louis, MO.

82. Wideman, R.F., Fedde, M.R., Tackett, C.D. and Weigle, G.E., 2000. Cardio-pulmonary function in preascitic (hypoxemic) or normal broilers inhaling ambient air or 100\% oxygen, Poultry Science, 79, 415-425.

83. Wideman, R.F., Rhoads, D.D., Erf, G.F. and Anthony, N.B., 2013. Pulmonary arterial hypertension (ascites syndrome) in broilers: a review, Poultry Science, 92, 64-83.

84. Xiang, R.P., Sun, W.D., Wang, J.Y. and Wang, X.L., 2002. Effect of vitamin C on pulmonary hypertension and muscularisation of pulmonary arterioles in broilers, British poultry science, $43,705-712$. 